TITLE:

\title{
Inverse Analysis of Experimental Scale Turbidity Currents Using Deep Learning Neural Networks
}

$\operatorname{AUTHOR}(S):$

Cai, Zhirong; Naruse, Hajime

\section{CITATION:}

Cai, Zhirong ... [et al]. Inverse Analysis of Experimental Scale Turbidity Currents Using Deep Learning Neural Networks. Journal of Geophysical Research: Earth Surface 2021, 126(8): e2021JF006276.

ISSUE DATE:

2021-08

URL:

http://hdl.handle.net/2433/268176

\section{RIGHT:}

(C2021. American Geophysical Union. All Rights Reserved.; The full-text file will be made open to the public on 19 February 2022 in accordance with publisher's 'Terms and Conditions for Self-Archiving'. 


\section{JGR Earth Surface}

\section{RESEARCH ARTICLE \\ 10.1029/2021JF006276 \\ Inverse Analysis of Experimental Scale Turbidity Currents Using Deep Learning Neural Networks}

Key Points:

- Inverse analysis of turbidity currents using deep learning neural networks was performed for experimental scale turbidites

- Inverse analysis results for numerical data sets proved that flow conditions can be reconstructed from characteristics of deposits

- Flow conditions and deposit profiles in flume experiments were also well reconstructed

Correspondence to:

Z. Cai,

cai.zhirong.64w@kyoto-u.jp

Citation:

Cai, Z., \& Naruse, H. (2021). Inverse analysis of experimental scale turbidity currents using deep learning neural networks. Journal of Geophysical Research: Earth Surface, 126, e2021JF006276. https://doi. org/10.1029/2021JF006276

Received 22 MAY 2021 Accepted 27 JUL 2021

(C) 2021. American Geophysical Union. All Rights Reserved.

\author{
Zhirong Cai ${ }^{1}$ (D) and Hajime Naruse ${ }^{1}$ (D) \\ ${ }^{1}$ Kyoto University, Kyoto, Japan
}

Abstract Despite the importance of turbidity currents in environmental and resource geology, their flow conditions and mechanisms are not well understood. This study proposes and verifies a novel method for the inverse analysis of turbidity currents using a deep learning neural network (DNN) with numerical and flume experiment data sets. Numerical data sets of turbidites were generated with a forward model. Then, the DNN model was trained to find the functional relationship between flow conditions and turbidites by processing the numerical data sets. The performance of the trained DNN model was evaluated with 2,000 numerical test data sets and five experiment data sets. Inverse analysis results on numerical test data sets indicated that flow conditions can be reconstructed from depositional characteristics of turbidites. For experimental turbidites, spatial distributions of grain size and thickness were consistent with the sample values. Concerning hydraulic conditions, flow depth, layer-averaged velocity, and flow duration were reconstructed with a certain level of deviation. The reconstructed flow depth and duration had percent errors less than $36.0 \%$ except for one experiment, which had an error of $193 \%$ in flow duration. The flow velocity was reconstructed with percent errors $2.38 \%-73.7 \%$. Greater discrepancies between the measured and reconstructed values of flow concentration $(1.79 \%-300 \%)$ were observed relative to the former three parameters, which may be attributed to difficulties in measuring the flow concentration during experiments. Although the DNN model did not provide perfect reconstruction, it proved to be a significant advance for the inverse analysis of turbidity currents.

Plain Language Summary This study performed inverse analysis on turbidity currents using a machine learning method. Flume experiments were conducted to verify the method. Turbidite, the deposit of turbidity current, is an active area of study because it is closely related to the exploration of petroleum resources. Since turbidites are often deposited as a result of tsunami events, the understanding of turbidity currents can also contribute to geohazard prevention. The inverse analysis method proposed in this study can help enhance our understanding of the flow properties of turbidity currents.

\section{Introduction}

A turbidity current is a process of sediment transport into subaqueous environments such as deep lakes and oceans (Daly, 1936; Johnson, 1939). Turbidites, the deposits of turbidity currents, are often characterized by graded bedding and sedimentary successions called the Bouma sequence (e.g., Bouma, 1962; Kuenen \& Migliorini, 1950; Talling et al., 2012). Turbidites have been an active area of study due to their close association with petroleum resources and their role in the destruction of sea-floor equipment, such as submarine cables (Talling et al., 2015; Weimer \& Slatt, 2007). Furthermore, turbidites are often deposited as a result of tsunami triggered turbidity currents (Arai et al., 2013) and thus can contribute to determine the recurrence intervals of geohazards.

Studying the flow behavior of turbidity currents is essential for understanding the characteristics of turbidites and their implications (Talling et al., 2007). However, knowledge in this area remains limited because of the difficulties in the direct observation of turbidity currents. Several in-situ measurements have been conducted (e.g., Arai et al., 2013; Paull et al., 2018; Vangriesheim et al., 2009; Xu et al., 2004) and extensive research detailing the dynamics of the measured flows was conducted (e.g., Azpiroz-Zabala et al., 2017; Chikita, 1989; Dorrell et al., 2016; Heerema et al., 2020; Simmons et al., 2020). However, measurements of hydraulic conditions, such as sediment concentration, were difficult because of the destructive nature and unpredictable occurrences of turbidity currents (Falcini et al., 2009; Lesshafft et al., 2011; Naruse \& Olariu, 2008; Talling et al., 2015). Recently, Simmons et al. (2020) proposed a novel acoustic method for 
measuring the concentration structure within submarine turbidity currents. The method was able to extract the sediment concentration data from ADCP measurements, but did not perform well at high concentrations. The method also assumed a single grain-size class in flow, which is not consistent with acutal flow in nature. Therefore, inverse analysis that reconstructs the flow conditions of turbidity currents from their deposits is crucial for estimating the flow conditions in natural environments.

Before this research, inverse analysis of turbidity currents was conducted by Baas et al. (2000), where flow velocity was reconstructed through analyses of sedimentary structures of turbidites. The results provided an estimation of the hydraulic conditions of flow at a single location, but did not provide a reconstruction of the spatial evolution of the turbidity current. In contrast, inverse analysis methods in previous studies based on numerical models provided more detailed insights into the spatial structure and evolution of flows over time (e.g., Falcini et al., 2009; Lesshafft et al., 2011; Parkinson et al., 2017). The method proposed by Falcini et al. (2009) assumed steady flow conditions and was simplified for obtaining analytical solutions, preventing it from accurately illustrating the flow mechanism of unsteady turbidity currents that can produce normally graded bedding. Consequently, this method cannot be applied to normally graded beds, which are typical characteristics of turbidites. Other studies used the optimization method, where the hydraulic parameters were determined by optimizing the input parameters of numerical models, so that the resulting calculations were consistent with the observed data from turbidites (Lesshafft et al., 2011). This method can provide a relatively good reconstruction of the hydraulic conditions of turbidity currents, but has an extremely heavy calculation load due to the complexity of the forward model employed and the repetitive calculation of the forward model for optimization. Therefore, applying the method to natural scale turbidites, which typically run over tens to hundreds of kilometers and flow continuously for several hours (Talling et al., 2015), is impossible. Optimization using the adjoint approach proposed by Parkinson et al. (2017) solved the problem of heavy calculation load, but the reconstructed values differed from the expected values up to an order of magnitude.

Since previous methods to estimate flow conditions for turbidites were either overly simplified (Baas et al., 2000), incapable of reproducing graded beds (Falcini et al., 2009), accurate but computationally intractable for natural scale turbidity currents (Lesshafft et al., 2011), or low in accuracy (Parkinson et al., 2017), a method that is both accurate and not computationally intractable should be developed. To resolve the aforementioned issues, Naruse and Nakao (2020) proposed a new method for inverse analysis of turbidite deposits using deep learning neural networks (DNN). A DNN model is a machine-learning computing system that works as a universal function approximator (Liang \& Srikant, 2016), meaning that an unknown function governing the relationship between observations within a domain is explored and approximated. Previously, it was applied to problems such as landslide susceptibility analyses (Pradhan et al., 2010) and identification of lithology from well log data (Rogers et al., 1992), where the empirical relationship between the observed data and parameters aimed to be predicted was explored. In the case of turbidity currents, however, it is impossible to obtain sufficient data sets of in-situ measurements of flow characteristics for developing a DNN inverse model. Instead of using in-situ measurements of turbidity currents in nature, Naruse and Nakao (2020) generated numerical data sets of turbidites using a forward model. The generated data sets were input into a DNN model to explore the functional relationship between turbidites and initial flow conditions. After this network training process, the DNN model can estimate flow conditions from new turbidite data. Naruse and Nakao (2020) performed inverse analysis using a trained DNN model on field scale numerical test data sets generated by a forward model. Their results showed that the DNN model can reconstruct flow properties from numerical test data sets and was robust against noise in input data. Although the DNN model has demonstrated its performance on numerical data sets, it is yet to be tested with turbidite data from experiments or in-situ measurements.

In this study, we verified the ability of the DNN model to perform inverse analysis of turbidity currents by applying it to the data collected from turbidites deposited in flume experiments. We chose to first test the DNN inverse model on flume experiments instead of field data, because turbidity currents were generated in a controlled environment during flume experiments. Conditions, including flow duration and initial hydraulic conditions, can be set manually, and measurements of these parameters can also be conducted easily during experiments. 


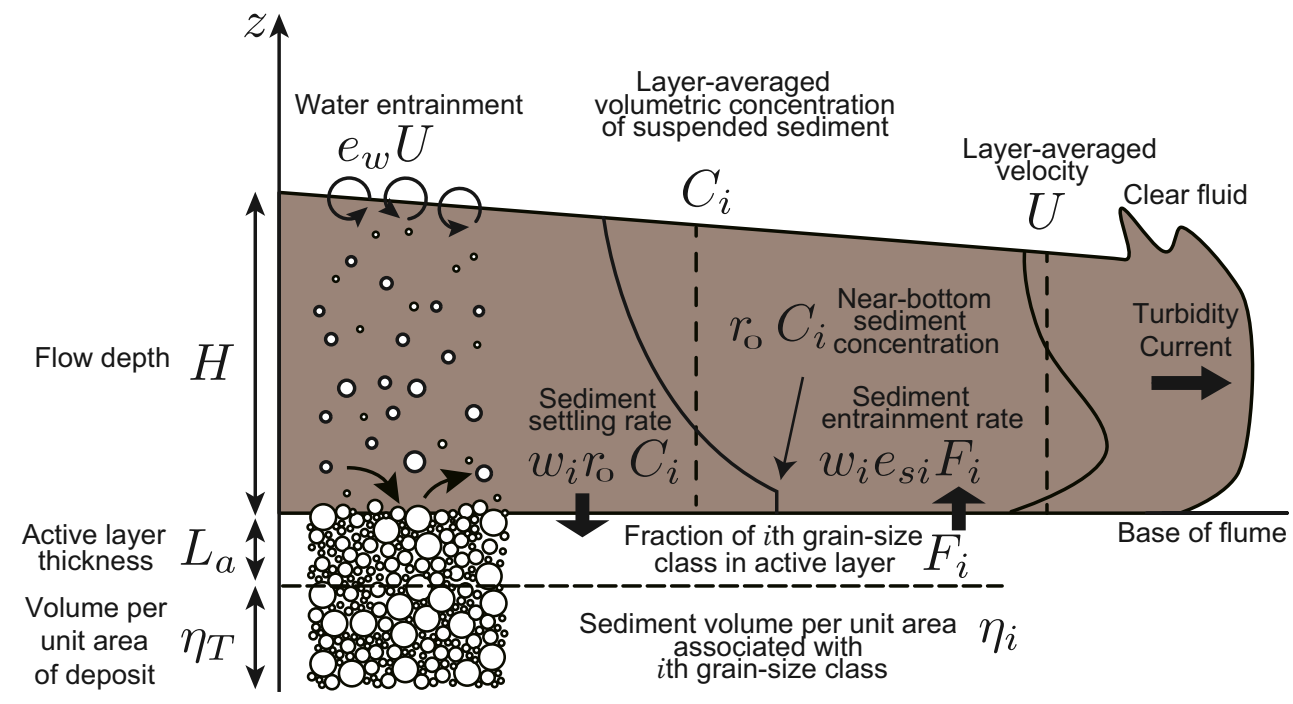

Figure 1. Schematic diagram of processes considered in the forward model from Nakao et al. (2020).

Here, we implemented a forward model and a DNN inverse model. The forward model was implemented with the same governing Equations as Naruse and Nakao (2020), but the numerical scheme and closure Equations were modified to accommodate experimental scale simulations and improve the accuracy of the calculation. The DNN model was trained with the experimental scale numerical data sets. The trained DNN model was first tested with independent sets of numerical data sets that were also produced by the forward model. Then, the trained DNN model was tested with flume experiment data. Initial flow conditions of experiments were reconstructed from sampled deposits. These flow conditions were then fed into the forward model to reconstruct the spatio-temporal evolution of the experiment. Reconstructed hydraulic conditions during the flow and grain size distribution of the deposits were compared with the measured values.

\section{Forward Model}

\subsection{Governing Equations}

The forward model implemented in this study is a layer-averaged shallow water model based on Kostic and Parker (2006). It is expanded to account for the transport and deposition of nonuniform grain size distribution discretized to multiple grain-size classes in Nakao et al. (2020) (Figure 1). This model was chosen because it is sufficiently complex to some details of the internal structure of flow, but also contains simplifications that make its calculation cost reasonable. The five governing Equations are as follows:

$$
\begin{gathered}
\frac{\partial H}{\partial t}+U \frac{\partial H}{\partial x}=e_{\mathrm{w}} U-H \frac{\partial U}{\partial x} \\
\frac{\partial U}{\partial t}+U \frac{\partial U}{\partial x}=R C_{\mathrm{T}} g\left(\sin \theta-\cos \theta \frac{\partial H}{\partial x}\right)-\frac{1}{2} g H R \cos \theta \frac{\partial C_{\mathrm{T}}}{\partial x}-\frac{U^{2}}{H}\left(c_{\mathrm{f}}-e_{\mathrm{w}}\right), \\
\frac{\partial C_{i}}{\partial t}+U \frac{\partial C_{i}}{\partial x}=\frac{w_{i}}{H}\left(F_{i} e_{\mathrm{s} i}-r_{\mathrm{o}} C_{i}\right)-\frac{e_{\mathrm{w}} C_{i} U}{H}, \\
\frac{\partial \eta_{i}}{\partial t}=\frac{w_{i}}{1-\lambda_{\mathrm{p}}}\left(r_{\mathrm{o}} C_{i}-e_{\mathrm{si}} F_{i}\right),
\end{gathered}
$$




$$
\frac{\partial F_{i}}{\partial t}+\frac{F_{i}}{L_{\mathrm{a}}} \frac{\partial \eta_{\mathrm{T}}}{\partial t}=\frac{w_{i}}{L_{\mathrm{a}}\left(1-\lambda_{\mathrm{p}}\right)}\left(r_{\mathrm{o}} C_{i}-e_{\mathrm{s} i} F_{i}\right),
$$

where the Equations represent fluid mass conservation (Equation 1), momentum conservation (Equation 2), sediment mass conservation (Equation 3), mass conservation in bed (Exner's Equation) (Equation 4), and sediment mass conservation in active layer (Equation 5) (Nakao et al., 2020).

Let $x$ and $t$ be the bed-attached streamwise coordinate and time, respectively. Parameters $H, U$, and $C_{i}$ represent the flow depth, the layer-averaged velocity, and the layer-averaged volumetric concentration of suspended sediment of the $i$ th grain-size class, respectively. In this study, the number of grain-size classes and representative grain diameters were determined on the basis of the grain size distribution in each experiment (specific values noted in Section 5.1). Parameter $C_{\mathrm{T}}$ denotes the layer-averaged total concentration of suspended sediment $\left(C_{\mathrm{T}}=\sum C_{i}\right)$, and $g$ represents gravitational acceleration. Parameter $c_{\mathrm{f}}$ is the friction coefficient. Parameter $\theta$ is the angle of inclination of the base slope. Sediment properties are described by $R$, the submerged specific density of sediment; $w_{i}$ represents the settling velocity of a sediment particle of the $i$ th grain-size class; $\lambda_{\mathrm{p}}$ represents the porosity of bed sediment. Parameter $\eta_{i}$ is the volume per unit area of bed sediment of the $i$ th grain-size class, and $\eta_{\mathrm{T}}$ is the sum of all $\eta_{i}\left(\eta_{\mathrm{T}}=\sum \eta_{i}\right)$. Parameters $L_{\mathrm{a}}$ represents the active layer thickness, and $F_{i}$ represents the volume fraction of the $i$ th grain-size class in active layer. Parameters $e_{\mathrm{s} i}, e_{\mathrm{w}}$, and $r_{\mathrm{o}}$ represent the entrainment rate of sediment of the $i$ th grain-size class into suspension, the entrainment rate of ambient water to flow, and the ratio of near-bed suspended sediment concentration to the layer-averaged concentration of suspended sediment, respectively (Figure 1).

\subsection{Closure Equations}

Empirical formulations from previous studies are adapted to close the governing Equations. In this study, the friction coefficient $c_{\mathrm{f}}$ is assumed to be a constant value. The particle settling velocity $w_{i}$ for each grainsize class with a representative grain diameter $D_{i}$ is calculated using the relation from Dietrich (1982), which can be expressed as follows:

$$
\begin{gathered}
w_{i}=R_{\mathrm{f} i} \sqrt{R g D_{i}}, \\
R_{\mathrm{f} i}=\exp \left(-b_{1}+b_{2} \log \left(R e_{\mathrm{p} i}\right)-b_{3}\left(\log \left(R e_{\mathrm{p} i}\right)\right)^{2}-b_{4}\left(\log \left(\operatorname{Re} e_{\mathrm{p} i}\right)\right)^{3}+b_{5}\left(\log \left(\operatorname{Re} e_{\mathrm{p} i}\right)\right)^{4}\right), \\
R e_{\mathrm{p} i}=\frac{\sqrt{\operatorname{Rg} D_{i}} D_{i}}{v},
\end{gathered}
$$

where $b_{1}, b_{2}, b_{3}, b_{4}$ and $b_{5}$ are 2.891,394, 0.95296, 0.056835, 0,000245, and 0.000245, respectively. $e_{\mathrm{w}}$ is calculated using the empirical formula from Fukushima et al. (1985) as follows:

$$
e_{\mathrm{w}}=\frac{0.00153}{0.0204+R i}
$$

with $R i$, the bulk Richardson number, defined as:

$$
R i=\frac{\operatorname{Rg} C_{\mathrm{T}} H}{U^{2}} .
$$

The entrainment coefficient of sediment $e_{\mathrm{s}}$ is calculated using the empirical relation from Garcia and Parker (1993):

$$
\begin{gathered}
e_{\mathrm{s} i}=\frac{a Z^{5}}{1+(a / 0.3) Z^{5}}, \\
Z=\alpha_{1} \frac{u_{*}}{w_{i}} R e_{\mathrm{p}}^{\alpha_{2}},
\end{gathered}
$$


where shear velocity $u_{*}$ is calculated as follows:

$$
u_{*}=\sqrt{c_{\mathrm{f}}} U,
$$

and the constants $\alpha_{1}$ and $\alpha_{2}$ are 0.586 and 1.23, respectively, if $R e_{\mathrm{p}} \leq 2.36$. If $R e_{\mathrm{p}}>2.36, \alpha_{1}$ and $\alpha_{2}$ are 1.0 and 0.6 , respectively. Constant $a$ is $1.3 \times 10^{-7}$. Kinematic viscosity of water $v$ is calculated as follows:

$$
v=\mu / \rho,
$$

where $\rho$ and $\mu$ denote water density and dynamic viscosity, respectively. The experimentally determined values for $\mu$ at $20.0^{\circ} \mathrm{C}$ (Rumble, 2018) were used in the calculation of $v$ in this study.

\subsection{Implementation of Forward Model}

In this study, the constrained interpolation profile (CIP) method (Yabe et al., 2001) implemented with staggered grid was used for integrating of the partial differential Equations 1, 2 and 3. The stability condition of the CIP scheme is as follows (Gunawan, 2015):

$$
1>\frac{\Delta t \max (|U|+\sqrt{g H})}{\Delta x} .
$$

In this study, the time step $\Delta t$ was fixed to a value of $0.01 \mathrm{~s}$ so that it does not violate the stability condition. The CIP scheme implemented was of third order accuracy. Although this numerical scheme is not strictly mass-conservative, the volume loss of this method has been verified to be less than $0.07 \%$ when tested with a simple numerical wave tank (NWT), acceptable for fluid simulation (Vestbøstad et al., 2007). To stabilize the calculation, artificial viscosity was used with the scheme of Jameson et al. (1981), where the parameter $\kappa$ was set to 0.25 . The two-step Adams predictor-corrector method, which was more stable than the ordinary Euler's method, was used to solve ordinary differential Equations 4 and 5. Interval of spatial grids $\Delta x$ was set to $0.05 \mathrm{~m}$ based on experimental settings (Section 4.1). The model was tested with different mesh sizes ranging from one fifth to five times the current mesh size and was confirmed to be mesh independent. Initial values of $\theta$ for all grids were set to the same value as the base slope of experimental setups.

The Dirichlet boundary condition was used for the upstream boundary, where all flow parameters at the upper boundary of the calculation domain, including the initial flow depth $H_{0}$, the initial flow velocity $U_{0}$, the initial total volumetric concentration of sediment $C_{\mathrm{T}, 0}$, and the initial volumetric concentration of each grain-size class $C_{i, 0}$, were set to be constant. Parameter $F_{i, 0}$, the initial volume fraction of the $i$ th grain-size class in active layer, was set to $1 / \mathrm{N}$ for all grain-size classes, where $\mathrm{N}$ represents the number of grain-size classes. The downstream boundary was the Neumann boundary condition in which all parameters were set to the same values as those of the grid adjacent to the lower boundary toward the upstream direction. Other than the upstream boundary, all flow parameters were initialized to zero. The wet-dry boundary condition at the head of the flow was conducted using the scheme proposed by Yang et al. (2016). A threshold value of $C_{\mathrm{T}} H, \epsilon$, was used to determine the position of the waterfront. If $C_{\mathrm{T}} H<\epsilon$, the grid was dry. If $C_{\mathrm{T}} H \geq \epsilon$, the grid was wet. In this study, $\epsilon$ was set to 0.000001 . A dry grid adjacent and downstream to a wet grid was a partial wet grid. Flow discharge $M$ at a partial wet grid $j$ was calculated using Homma's Equation (Yang et al., 2016) as follows:

$$
M=C_{\mathrm{s}} H_{j-1} \sqrt{\operatorname{Rg} C_{\mathrm{T}, j-1} H_{j-1}},
$$

where $C_{s}$, the discharge coefficient, is equal to 0.35 .

The density of the surrounding fluid $\rho$ was set to $1,000.0 \mathrm{~kg} / \mathrm{m}^{3}$ in this study, since experiments were conducted with water. The submerged specific density of sediment $R=\left(\rho_{s}-\rho\right) / \rho$ was set differently according to the types of particles used in experiments ( $\rho_{s}$ is the density of sediment particles), which are stated in Section 4.1. The porosity of bed sediment $\lambda_{\mathrm{p}}$ was assumed to be 0.4 . In this study, both the friction coefficient $c_{\mathrm{f}}$ and ratio of near-bed concentration to layer-averaged values $r_{\mathrm{o}}$ were assumed to be constant. $c_{\mathrm{f}}$ was set to 0.004. $r_{\mathrm{o}}$ was set to 1.5 (Kostic \& Parker, 2006). In addition, the thickness of active layer $L_{\mathrm{a}}$ was set to be a constant, $0.003 \mathrm{~m}$ (Arai et al., 2013). The gravitational acceleration $g$ was $9.81 \mathrm{~m} / \mathrm{s}^{2}$. 


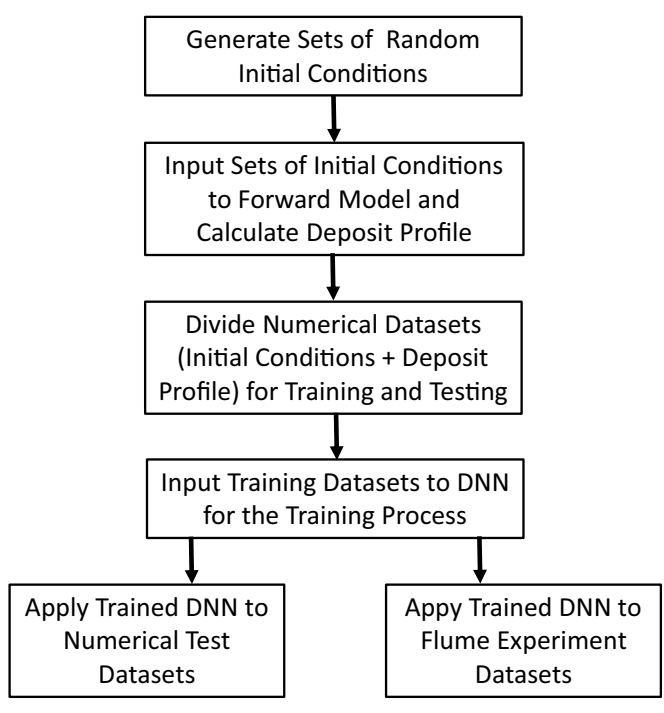

Figure 2. A flowchart illustrating the procedures from generation of numerical data to the application of a deep learning neural network model to numerical test data sets and flume experiment data sets.

\section{Inverse Analysis by Deep Learning Neural Network}

In this method, initial flow conditions of turbidity currents are reconstructed from their turbidite deposits. The DNN model first explores the functional relationship between the initial flow conditions of turbidity currents and the resulting turbidite deposits via training. After training, the DNN model is applied to new turbidite data sets for inverse analysis. In preparation for training, numerical training data sets are generated using the forward model. During training, the training data sets are fed into the DNN. The DNN model examines the data sets and adjusts its internal parameters to make a good estimation of the initial flow conditions from the deposit profile. After training, the DNN, which can predict the initial flow conditions of new turbidites based on the functional relationship it discovered, is tested with independent numerical data sets that are also generated by the forward model and with flume experiment data. The procedure of using the DNN model as a method of inverse analysis in this study is illustrated in a flowchart in Figure 2.

\subsection{Generation of Training Data}

A training data set is a combination of randomly generated initial flow conditions at the upstream boundary of the flow and a matching deposit profile calculated using the forward model. A program in Python was written to generate sets of initial flow conditions. Each set of flow conditions generated consists of an initial flow velocity $U_{0}$, an initial flow depth $H_{0}$, a flow duration $T_{\mathrm{d}}$, and the initial concentrations of each grain-size class $C_{i, 0}$. Other variables, such as slope, are set to constant values. The slope was set according to values of slope in experiments conducted (Section 4.1).

The forward model calculates the deposit profile of a turbidite using randomly generated initial flow conditions. The deposit profile is calculated as volume per unit area for each grain-size class at 60 locations within a $3 \mathrm{~m}$ range downstream from the upstream boundary. Each data point is $0.05 \mathrm{~m}$ away from its neighboring points. These data points are akin to sampled data from flume experiments or core or outcrop data from actual turbidites. Since fewer data points can be obtained from experiments or actual turbidites, details of deposit profiles need to be interpolated from available data points. Table 1 illustrates the ranges of randomly generated initial flow conditions. These ranges were decided on the basis of possible values that can be observed in experimental scale turbidity currents. Since terms in the forward model calculation were set to be consistent with experimental settings instead of natural scale turbidity currents, no range of values beyond that of experimental scale would be appropriate for the current model implemented. In this study, 10,000 training data sets were used for training and 2,000 data sets were used for verifying the DNN. The number of test data sets was chosen to be the same number as that of validation data sets. The test numerical data sets for verification were generated independently from the training data sets.

Table 1

Range of Initial Flow Conditions Generated for the Generating of Training Data Sets

\begin{tabular}{lcc}
\hline Parameter & Minimum & Maximum \\
\hline$H_{0}(\mathrm{~m})$ & 0.01 & 0.3 \\
$U_{0}(\mathrm{~m} / \mathrm{s})$ & 0.01 & 0.2 \\
$C_{i, 0}$ & 0.0002 & 0.005 \\
$T_{\mathrm{d}}(\mathrm{s})$ & 180 & 1,080 \\
\hline
\end{tabular}


(a)

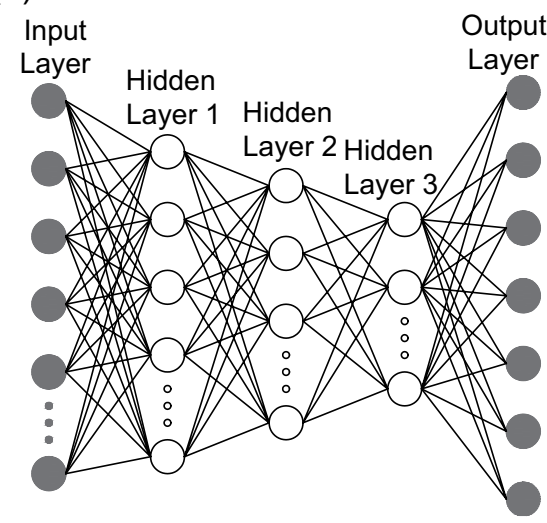

(b)

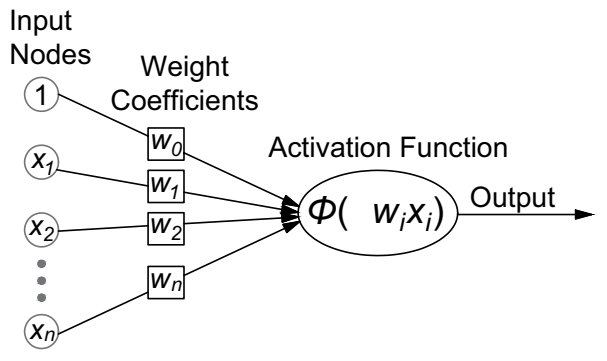

Figure 3. Schematic diagrams of deep learning neural network (DNN). (a) Overall structure of DNN. (b) Concept of weight coefficient and activation function.

\subsection{Structure of Deep Learning Neural Network}

The type of neural network (NN) used in this study is a fully connected NN, which consists of an input layer, several hidden layers, and an output layer. Each layer consists of some nodes. Each node connects with every node in the adjacent layers (Figure 3a). Nodes in the input layer hold deposit profile values, that is, the volume-per-unit-area for all grain-size classes at spatial grids. Nodes in the output layer hold estimates of parameters we seek to reconstruct, which in this case are the initial flow conditions $U_{0}, H_{0}, C_{i, 0}$, and the flow duration $T_{\mathrm{d}}$. The activation function used in this study is the rectified linear unit (ReLU), which is one of the most commonly used activation functions for DNNs and is proven to perform calculations at a higher speed than other activation functions (Krizhevsky et al., 2012).

Before training, the weight coefficients are set to random values. As the training process begins, deposit profile values from the training data sets are fed into the input layer. These values propagate through the hidden layers of the DNN, and estimates of the initial flow conditions are outputted at the output layer. At this point in the training process, the DNN model is yet to adapt its internal variables to the functional relationship between turbidite deposits and initial flow conditions. Thus, the initial estimates are expected to largely differ from the actual values. To explore this functional relationship, a loss function is used to evaluate the accuracy of the estimated values. The loss function used in this case is the mean squared error function, which is considered as one of the best functions for regression (Wang \& Bovik, 2009). The gradient of the loss function is calculated and fed back to the hidden layers of the DNN model through backpropagation (Nielsen, 2015; Schmidhuber, 2015), where the internal values of the DNN model are optimized toward minimizing the difference between the estimated and actual values. This process is repeated for every epoch of calculation. An epoch is a cycle of calculation in a DNN that involves one forward pass and one backpropagation of all training data.

The optimization algorithm used in this study is stochastic gradient descent (SGD), which drastically reduces the amount of calculation involved in training without compromising accuracy compared to previous gradient descent algorithms (Bottou, 2010). In this study, Nesterov momentum is used with SGD (Ruder, 2016). Because of the difference in the order of the range of the initial flow conditions, the training data sets should be normalized before they are inputted to the DNN. In this case, all values are normalized to be between 0 and 1 for the DNN model to consider all parameters at equal weights. The hyperparameters, including the number of layers, number of nodes at each layer, dropout rate, validation split, learning rate, batch size, epoch, and momentum, were adjusted manually. Various combinations were attempted. The best combination of hyperparameters was chosen on the basis of the performance of the DNN, which is judged on the basis of the final validation loss.

In this study, the DNN model was developed using Python with the package Keras 2.2.4. The package Tensorflow 1.14.0 (Abadi et al., 2015) was used for backend calculations. Calculations were performed using GPU NVIDIA GeForce GTX 1080 Ti. 


\subsection{Evaluation of Trained DNN Model}

During the verification of the DNN model with test numerical data sets (Section 5.1), the reconstruction result of each parameter was evaluated using bias $(B)$ and sample standard deviation $(s)$ of residuals. The calculations were performed using the following Equations:

$$
\begin{gathered}
B=\frac{\sum x_{i}}{n}, \\
s=\sqrt{\frac{\sum\left(x_{i}-B\right)^{2}}{n-1}},
\end{gathered}
$$

where $n$ represents the number of test data sets, and $x_{i}$ denotes the residual of the specific reconstructed parameter for the $i$ th test data set. The value of $s$ for each reconstructed parameter was compared with a representative value $C_{v}^{*}$, which is the mid-value over the range in which the specific parameter was generated (Table 1). The confidence interval of $B$ was determined using the bootstrap resampling method (Davison \& Hinkley, 1997). Resampling of $B$ was conducted 10,000 times, and the $95 \%$ confidence interval (CI) of $B$ was determined.

During the verification of the DNN model using flume experiment data (Section 5.2), linear interpolation was first applied to the sampled experimental deposit data sets so that the number of data points for one experimental data set was the same as that for a training data set. Then, flow parameters at the upstream end of the simulation were reconstructed from the measured properties of the deposit profile. The upstream end of the simulation was set at $1.0 \mathrm{~m}$ from the inlet of the flume. The reconstructed parameters were inputted into the forward model so that downstream flow parameters and the time evolution of the deposit profile were calculated. The calculated downstream flow parameters were compared with the flow conditions measured during experiments. The deposit profile calculated from the reconstructed flow parameters was also compared with the measured deposit profile that was used for inversion.

To evaluate the precision of reconstruction, Jackknife method (McIntosh, 2016) was applied to the sampled deposit values and delete-1 Jackknife samples were generated. Inverse analysis by the DNN model was performed for the delete-1 Jackknife samples, and downstream flow parameters were calculated for each sample. There were 18 delete- 1 Jackknife samples for each experiment, since the deposits were sampled at 18 locations. Considering the small sample size (less than 30), t-distribution was used instead of normal distribution. The $95 \%$ confidence interval of t-distribution is $\pm\left(t \times s_{\bar{x}}\right)$, where $s_{\bar{x}}$ is the standard error and is defined by the following Equations:

$$
\begin{gathered}
\bar{x}=\frac{\sum_{i=1}^{n} x_{i}}{n}, \\
s_{\bar{x}}=\sqrt{\frac{\sum_{i=1}^{n}\left(x_{i}-\bar{x}\right)^{2}}{n(n-1)}},
\end{gathered}
$$

where $n$ represents the sample size, $x_{i}$ denotes the jackknife sample where the $i$ th sampled deposits value were eliminated, and $\bar{x}$ is the mean of $x_{i}$. The value $t$ is a standardized value determined by the degree of freedom and the alpha level. Degree of freedom is the sample size subtracted by 1 . In this case, the sample size is 18 , thus $\mathrm{df}$ is 17 . For $95 \%$ confidence interval, the alpha level is 0.05 . According to the two-tails t-distribution table, $t$ for our samples is 2.110 .

\section{Flume Experiments}

\subsection{Experiment Settings}

The flume was made of acrylic panels and was $4 \mathrm{~m}$ in length, $0.12 \mathrm{~m}$ in width, and $0.5 \mathrm{~m}$ in depth. During the experiments, it was submerged in a tank made of glass panels and a steel supporting frame. The tank 


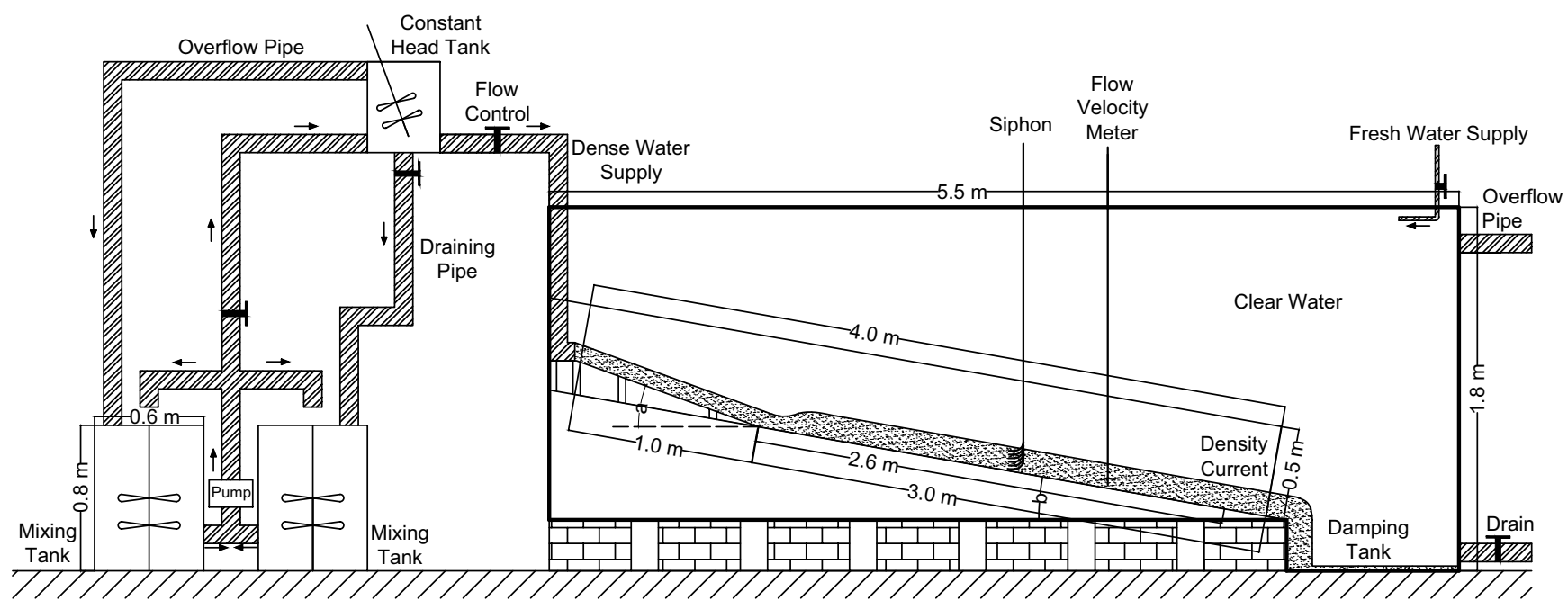

Figure 4. Schematic diagram of flume used for experiment.

was $5.5 \mathrm{~m}$ in length, $2.5 \mathrm{~m}$ in width, and $1.8 \mathrm{~m}$ in depth. The slope of the channel floor changed at $1.0 \mathrm{~m}$ from the inlet, where $a$ was the upstream slope and $b$ was the downstream slope (Figure 4). Values of $a$ and $b$ for each experiment are stated in Table 2.

Sediment was mixed with water in two mixing tanks before the experiments. During the experiments, the mixture of sediment and water was first pumped to the constant head tank and then released into the flume. The flow into the flume was controlled via a valve at the base of the constant head tank. Flow discharge was regulated by changing the degree of valve opening. The amount of mixture in the constant head tank was kept at a constant level during the experiments to maintain a stable flow discharge. The damping tank at the downstream end of the flume prevented any reflection of flow toward the upstream direction. A pipe of freshwater supply was placed at the top of the damping tank, and a draining pipe was placed at the bottom of the damping tank. The combination of these two pipes kept the level of water in the tank constant and prevented the reflection of flow.

Five experiments were conducted using plastic particles in this study. The density of the plastic particles used was $1.45 \mathrm{~g} / \mathrm{cm}^{3}$. Two experiments (experiments PP1, PP2) were performed using polyvinyl

Table 2

Conditions and Settings of Experiments Conducted

\begin{tabular}{lccccc}
\hline & PP1 & PP2 & PP3 & PP4 & PP5 \\
\hline$C_{\mathrm{TT}}$ & 0.0191 & 0.0276 & 0.0120 & 0.0141 & 0.0101 \\
$C_{1 \mathrm{~T}}$ & 0.0102 & 0.0160 & 0.00230 & 0.00453 & 0.00290 \\
$C_{2 \mathrm{~T}}$ & 0.00713 & 0.00820 & 0.00670 & 0.00657 & 0.00446 \\
$C_{3 \mathrm{~T}}$ & 0.00146 & 0.00254 & 0.00250 & 0.00246 & 0.00199 \\
$C_{4 \mathrm{~T}}$ & 0.000366 & 0.000817 & 0.000460 & 0.000567 & 0.000766 \\
$x_{\mathrm{C}}(\mathrm{m})$ & 1.08 & 2.10 & 1.50 & 1.50 & 1.50 \\
$x_{\mathrm{U}}(\mathrm{m})$ & 1.46 & 2.48 & 1.20 & 1.20 & 1.20 \\
$x_{\mathrm{H}}(\mathrm{m})$ & 1.10 & 1.10 & 1.20 & 1.20 & 1.20 \\
Temperature $\left({ }^{\circ} \mathrm{C}\right)$ & 22.5 & 17.0 & 13.0 & 13.5 & 14.0 \\
Slope $a$ & $26.8 \%$ & $26.8 \%$ & $25.6 \%$ & $25.6 \%$ & $25.6 \%$ \\
Slope $b$ & $10.0 \%$ & $10.0 \%$ & $8.00 \%$ & $8.00 \%$ & $8.00 \%$ \\
\hline
\end{tabular}


chloride, which had an average grain diameter of $0.120 \mathrm{~mm}$, and melamine, which had an average grain diameter of $0.220 \mathrm{~mm}$ (Section 4.3). Three experiments (experiments PP3, PP4, PP5) were performed with two types of melamine, which had an average grain diameter of $0.120 \mathrm{~mm}$ and $0.220 \mathrm{~mm}$, respectively (Section 4.3).

\subsection{Measurements and Data Analysis}

Before each experiment, the tank water temperature was measured using a glass alcohol thermometer. The mixture in the tank was sampled with a $500 \mathrm{~mL}$ beaker to measure the initial concentration in the tank. Flow velocity was measured using an acoustic Doppler velocity profiler (ADVP; Nortek Vectrino Profiler). The maximum functional range of the ADVP used was $4.0-7.0 \mathrm{~cm}$ below the probe. The actual range of reliable measurement may be shorter if the signal-to-noise ratio (SNR) of data collected is below a certain threshold (Appendix A). To obtain the vertical velocity profile of the flow, an actuator was used to adjust the position of the ADVP during the experiments.

A siphon with 10 plastic tubes was used to measure the suspended sediment concentration of the flow. The tubes were aligned vertically at $1.0 \mathrm{~cm}$ intervals and were positioned such that samples were collected at 0.0 to $9.0 \mathrm{~cm}$ above the bed. Aluminum tubes with an outer diameter of $8.0 \mathrm{~mm}$ and an inner diameter of 5.0 $\mathrm{mm}$ were attached to the outlets of plastic tubes to keep them in place. Sampling by siphon was conducted when the flow reached a quasi-equilibrium state. The state of flow was determined by observing the development of the flow. Two single-lens reflex cameras were used to record the experiments. Flow depth was determined based on the video recorded.

After the experiments, the flume was left untouched for 1-3 days for the suspended sediment to settle. Afterward, photos were taken from a lateral view perpendicular to the flume. The lateral view of the deposited sediment was photographed with a ruler beside it. The height of the deposit was determined from the photos. Water was then gradually drained from the tank with a bath pump at a rate of $0.0002333 \mathrm{~m}^{3} / \mathrm{s}$. After the water was drained, deposited sediment was sampled at $20 \mathrm{~cm}$ intervals starting from the upstream boundary of the flume.

Samples from the siphon and the mixing tank were first weighed immediately after they were collected. Then, they were dried in a drying oven at $70^{\circ} \mathrm{C}$ along with the deposit samples. Samples from the siphon and the mixing tank were weighed again after drying. The measurements were used for calculating the sediment concentration in the flow and tank. Grain size distribution analysis was performed in a settling tube for all dried sediment samples. The settling tube used was $1.8 \mathrm{~m}$ in length. The calculation of grain size distribution was performed using STube (Naruse, 2005). Particle settling velocity was calculated using Gibbs (1974). The measured grain size distribution of sediment was discretized to four grain-size classes. The representative grain diameter of grain-size classes 1, 2, 3, and 4 were set to be $210,149,105$, and $74.3 \mu$ $\mathrm{m}$, respectively.

In steady flow conditions, the relationship between the layer-averaged flow velocity $U$, the layer-averaged sediment volumetric concentration $C$, and the flow depth $H$ is defined as follows (Garcia \& Parker, 1993):

$$
U C H=\int_{a}^{\infty} u_{z} c_{z} d z
$$

where $u_{z}$ and $c_{z}$ represent the flow velocity and sediment volumetric concentration, respectively, at elevation $z$ above the bed. The relationship between the layer-averaged flow velocity $U$ and the velocity maximum $U_{\mathrm{m}}$ is defined by the following equation (Altinakar et al., 1996):

$$
\frac{U_{\mathrm{m}}}{U}=1.3 .
$$


The layer-averaged flow velocity was calculated from the velocity maximum of the profile measured by the ADVP using the relationship described by Equation 22. The sediment volumetric concentration was calculated from siphon measurements using the relationship described by Equation 21.

\subsection{Experimental Conditions}

Experimental conditions for the five runs conducted are outlined in Table 2. $C_{\mathrm{TT}}$ represents the total concentration of sediment in the mixing tank. $C_{1 \mathrm{~T}}, C_{2 \mathrm{~T}}, C_{3 \mathrm{~T}}$, and $C_{4 \mathrm{~T}}$ represent the concentrations of grain-size classes $1,2,3$, and 4, respectively. Parameter $x_{\mathrm{C}}$ represents the position of the siphon downstream, whereas $x_{\mathrm{U}}$ represents the position of ADVP downstream. $x_{\mathrm{H}}$ represents the position in which the flow depth was measured from a video taken during the experiments. $x_{\mathrm{U}}, x_{\mathrm{C}}$ and $x_{\mathrm{H}}$ were changed for each run because of limitations in the flume setup at the time of the experiments. Temperature is the measured temperature of clear water in the tank before the experiments.

\section{Results}

Inverse analysis was applied to deposits within a $2.6 \mathrm{~m}$ range downstream of the beginning of the slope $b$ (1.0 $\mathrm{m}$ from the inlet of flow). Due to the limited size of the flume, slope $a$ was set to a steep angle (26.8\% or $25.6 \%$ ) in all five experiments to ensure that the flow accelerates sufficiently for entrainment to occur. Considering the instabilities near the inlet and the overly steep slope, the region with slope $a$ was excluded from numerical simulations and inverse analysis. For the generation of numerical data sets, the upstream boundary of the simulation was set at the beginning of slope $b$, and calculations were performed for a $3.0 \mathrm{~m}$ range downstream. The actual sampling of experiment deposits was performed only up to $2.6 \mathrm{~m}$ from the beginning of slope $b$ (Figure 4), because deposits beyond the region were too thin to be collected for some experiments. Only simulation data from the same range were used for training and verification to match the actual sampling range of experiment deposits.

For hyperparameters used during training, the dropout rate, validation split, and momentum for the DNN model were set to $0.5,0.2$, and 0.9 , respectively. The learning rate was set to 0.01 . The batch size was set to 32 and the number of layers was set to 5. The number of nodes for each layer was 2,000. Epoch was 20,000. With this setting, the validation loss was 0.0033 for training with $10.0 \%$ slope data sets and 0.0038 for training with $8.00 \%$ slope data sets. Figures 5 and 6 a show that overlearning did not occur, as no deviation was observed between the resulting values of the loss functions for the training and validation data sets.

\subsection{Verification of Inverse Model With Test Numerical Data Sets}

This section presents the verification results with numerical test data sets. Parameter reconstruction results by the DNN model are shown in Figures 5 and 6 . Parameters reconstructed include flow duration $T_{\mathrm{d}}$ and flow conditions at the upstream end (flow velocity $U_{0}$, flow depth $H_{0}$, and sediment concentrations $C_{i, 0}$ ). Separate verification was performed with numerical data sets of experiments conducted with $10.0 \%$ slope and $8.00 \%$ slope. Verification results are described in Sections 5.1.1 and 5.1.2.

\subsubsection{Verification With Test Numerical Data Sets of Experiments Conducted With 10.0\% Slope}

Overall, the reconstructed values mostly matched with the original values, with a few outliers (Figures 5b$5 \mathrm{~h})$. However, a greater degree of scattering was observed for $T_{\mathrm{d}}$ compared with other parameters. $T_{\mathrm{d}}$ seemed to show a tendency of being underestimated (Figure $5 \mathrm{~b}$ ). The ranges of misfit ( $2 s)$ were reasonable for all parameters, which had $2 s / C_{v}^{*}$ values under $22.0 \%$ (Table 3). For $C_{i, 0}$, zero was within the $95 \% \mathrm{CI}$ of $B$, but not for $T_{\mathrm{d}}, U_{0}$, and $H_{0}$. CI range was below zero for $T_{\mathrm{d}}$ and $U_{0}$ and above zero for $H_{0}$.

\subsubsection{Verification With Test Numerical Data Sets of Experiments Conducted With $8.00 \%$ Slope}

Overall, good correlations were observed for the reconstructed and original values of flow parameters. The reconstructed values were mostly consistent with the original values, with a few outliers (Figures $6 \mathrm{~b}-6 \mathrm{~h}$ ). Similar to the test data sets described in Section 5.1.1, a tendency of underestimation was observed for $T_{\mathrm{d}}$ 
(a)

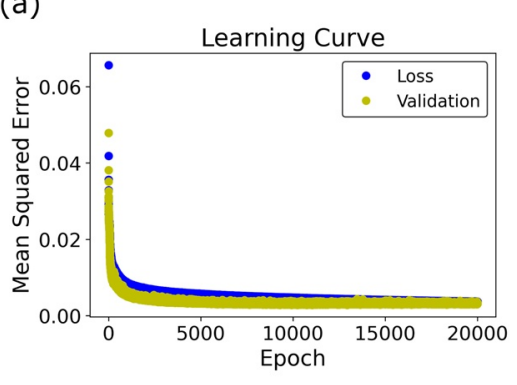

(b)

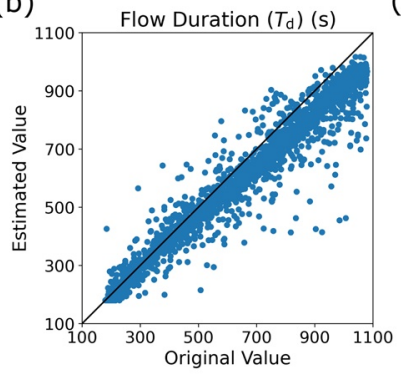

(c)

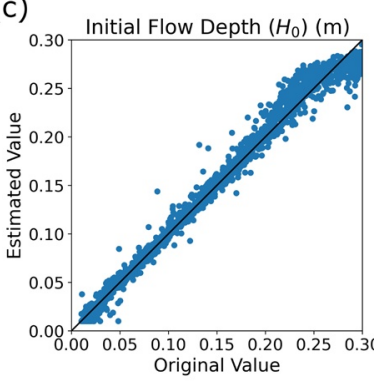

(d)

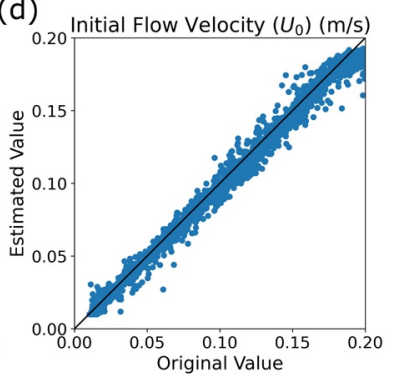

(e)

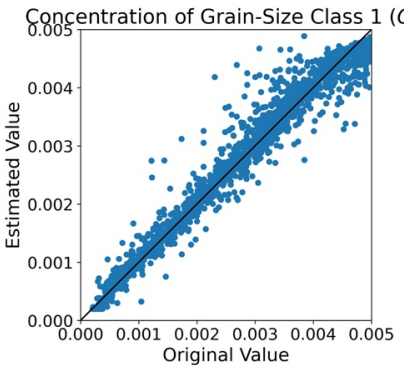

(f)

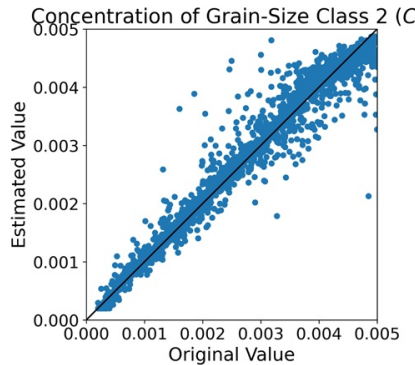

(

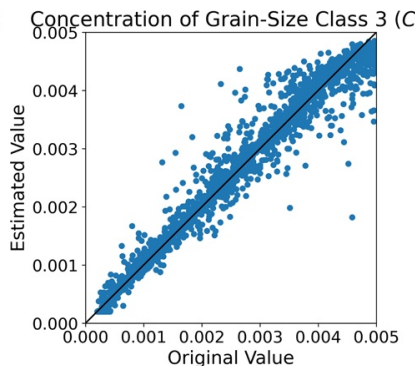

(h)

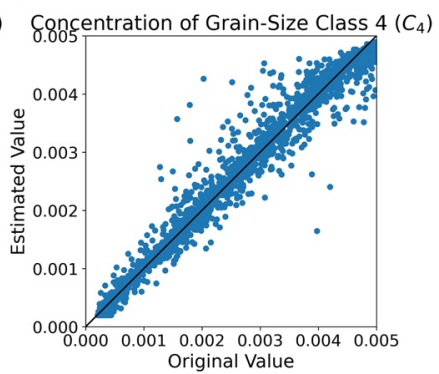

Figure 5. Results of verification with independent numerical data sets (slope $=10.0 \%$ ). The black diagonal line in each graph is where values on the $x$-axis (the true values) equal to the values on the $y$-axis (the estimated values). If a point lies on this line, the reconstructed value matches the true value perfectly. (a) Learning curve. (b) Estimates of $T_{\mathrm{d}}$. (c) Estimates of $H_{0}$. (d) Estimates of $U_{0}$. (e) Estimates of $C_{1,0}$. (f) Estimates of $C_{2,0}$. (g) Estimates of $C_{3,0}$. (h) Estimates of $C_{4,0}$.

(Figure 6b). The range of misfit (2s) was reasonable for all parameters, which had $2 s / C_{v}^{*}$ values under $23.0 \%$ (Table 4). Zero was included in the $95 \% \mathrm{CI}$ of $B$ for $U_{0}, C_{2,0}$, and $C_{3,0}$, but not for $T_{\mathrm{d}}, H_{0}, C_{1,0}$, and $C_{4,0}$. CI range was below zero for $T_{\mathrm{d}}$ and $H_{0}$ and above zero for $C_{1,0}$ and $C_{4,0}$.

(a)

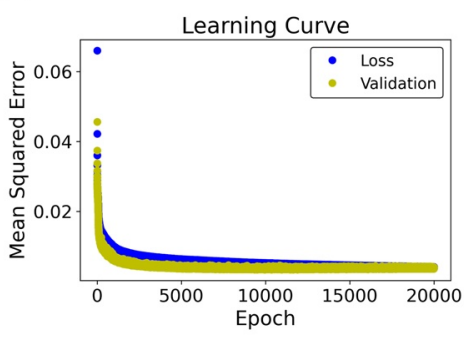

(e)

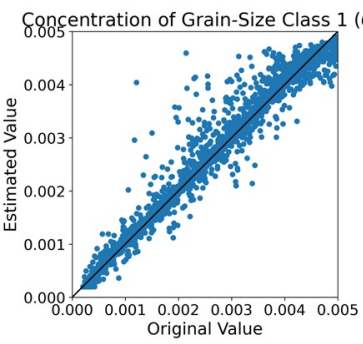

(b)

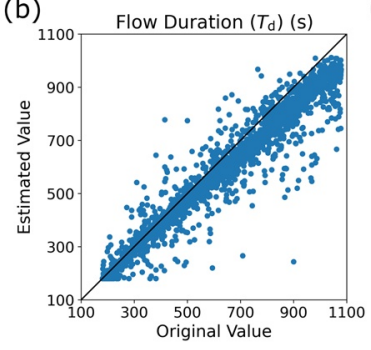

(f)

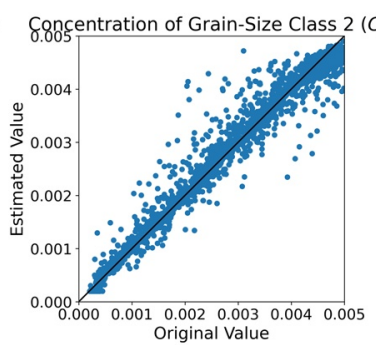

(g) (c)
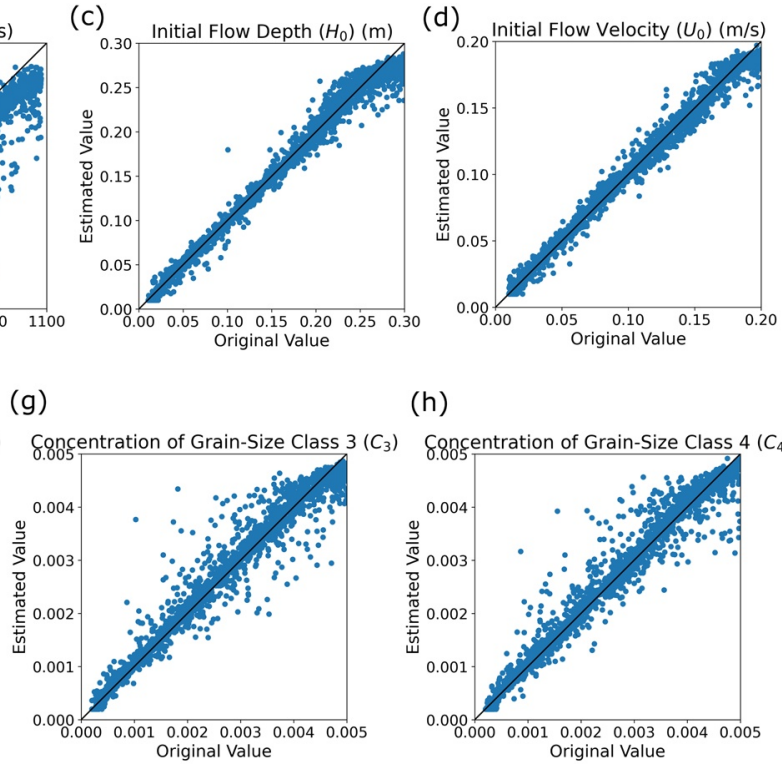

(h)

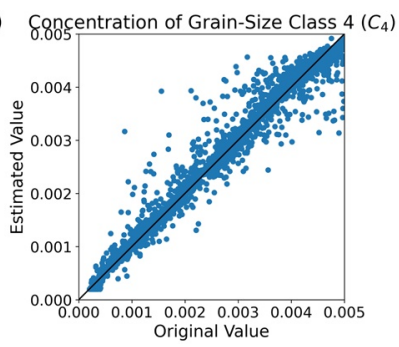

Figure 6. Results of verification with independent numerical data sets (slope $=8.00 \%$ ). The black diagonal line in each graph is where values on the $x$-axis (the true values) equal to the values on the $y$-axis (the estimated values). If a point lies on this line, the reconstructed value matches the true value perfectly. (a) Learning curve. (b) Estimates of $T_{\mathrm{d}}$. (c) Estimates of $H_{0}$. (d) Estimates of $U_{0}$. (e) Estimates of $C_{1,0}$. (f) Estimates of $C_{2,0}$. (g) Estimates of $C_{3,0}$. (h) Estimates of $C_{4,0}$. 
Table 3

Sample Standard Deviation and Bias of the Inversion Result for Numerical Data Sets of Experiments Conducted With 10.0\% Slope

\begin{tabular}{lccccc}
\hline Parameters & $s$ & $C_{v}^{*}$ & $2 s / C_{v}^{*}$ & \multicolumn{1}{c}{$B$} & CI of $B$ \\
\hline$U_{0}(\mathrm{~m} / \mathrm{s})$ & 0.00577 & 0.105 & 0.110 & -0.00234 & $(-0.00316,-0.00155)$ \\
$H_{0}(\mathrm{~m})$ & 0.00978 & 0.155 & 0.126 & 0.00164 & -49.4 \\
$T_{\mathrm{d}}(\mathrm{s})$ & 68.6 & 630 & 0.218 & $0.000286,0.00301)$ \\
$C_{1,0}$ & 0.000254 & 0.0026 & 0.195 & $0.0000318 \quad(-59.1,-40.1)$ & $(-0.00000234,0.0000679)$ \\
$C_{2,0}$ & 0.000278 & 0.0026 & 0.214 & 0.0000292 & $(-0.00000832,0.0000681)$ \\
$C_{3,0}$ & 0.000280 & 0.0026 & 0.215 & 0.0000149 & $(-0.0000237,0.0000536)$ \\
$C_{4,0}$ & 0.000271 & 0.0026 & 0.209 & 0.0000234 & $(-0.0000130,0.0000617)$ \\
\hline
\end{tabular}

\subsection{Inverse Analysis of Flume Experiment Data}

In this section, the calculated deposit profiles and grain size distributions are compared with the actual deposit profiles sampled from the experiments (Figures 7 and 8). The results of the reconstructed flow conditions, including flow velocity $U_{x_{U}}$, flow depth $H_{x_{H}}$, sediment concentrations $C_{x_{C}}$, and flow duration $T_{\mathrm{d}}$, are compared with the measured values (Tables 5 and 6). $H_{x_{H}}$ is the flow depth $H$ at position $x_{H}$ (see the positions in Table 2) downstream when the flow reached a quasi-equilibrium state. $U_{x_{U}}$ is the velocity $U$ at position $x_{U}$ downstream when the flow reached a quasi-equilibrium state. $C_{x_{C}}$ is the $C$ at position $x_{C}$ downstream when the flow reached a quasi-equilibrium state. Inverse analysis results of the experiments conducted with $10.0 \%$ slope (PP1, PP2) and 8.00\% slope (PP3, PP4, PP5) are described in Sections 5.2.1 and 5.2.2.

\subsubsection{Experiments Conducted With $10.0 \%$ Slope (PP1, PP2)}

Deposit profiles of experiments PP1 and PP2 demonstrated a thinning and fining downstream trend with concave-upward geometry. For both runs, the reconstructed deposit profiles of the total deposition almost entirely overlapped with the sampled data (Figures $7 \mathrm{a} 1$ and $7 \mathrm{~b} 1$ ). The reconstructed grain size distributions at $1.4 \mathrm{~m}$ and $1.8 \mathrm{~m}$ downstream also agreed well with the measured values for both experiments (Figures $7 \mathrm{a} 2,7 \mathrm{a} 3,7 \mathrm{~b} 2$ and $7 \mathrm{~b} 3)$.

For flow depth $H_{x_{H}}$, the measured and reconstructed values were in good agreement (Figure 9a). The measured $H_{x_{H}}$ of PP1 was $0.116 \mathrm{~m}$ and the reconstructed value was $0.157 \mathrm{~m}$ with an uncertainty range $(95 \%$ confidence interval) of $\pm 0.00921 \mathrm{~m}$. The measured $H_{x_{H}}$ of PP2 was $0.123 \mathrm{~m}$ and the reconstructed value was $0.142 \mathrm{~m}$ with anuncertainty range of $\pm 0.00849 \mathrm{~m}$. Reconstructed $H_{x_{H}}$ of both PP1 and PP2 had a relatively small uncertainty range in comparison to the measured and reconstructed values. The percent errors between reconstructed and measured $H_{x_{H}}$ were $35.1 \%$ and $15.3 \%$ for PP1 and PP2, respectively (Table 5). For

Table 4

Sample Standard Deviation and Bias of the Inversion Result for Numerical Data Sets of Experiments Conducted With 8.00\% Slope

\begin{tabular}{|c|c|c|c|c|c|}
\hline Parameters & $s$ & $C_{v}^{*}$ & $2 s / C_{v}^{*}$ & $B$ & CI of $B$ \\
\hline$U_{0}(\mathrm{~m} / \mathrm{s})$ & 0.00637 & 0.105 & 0.121 & 0.000369 & $(-0.000532,0.00124)$ \\
\hline$H_{0}(\mathrm{~m})$ & 0.0107 & 0.155 & 0.138 & -0.00225 & $(-0.00376,-0.000790)$ \\
\hline$T_{\mathrm{d}}(\mathrm{s})$ & 72.1 & 630 & 0.229 & -47.3 & $(-57.4,-37.5)$ \\
\hline$C_{1,0}$ & 0.000285 & 0.0026 & 0.219 & 0.0000538 & $(0.0000149,0.0000947)$ \\
\hline$C_{2,0}$ & 0.000279 & 0.0026 & 0.215 & 0.0000339 & $(-0.00000493,0.0000736)$ \\
\hline$C_{3,0}$ & 0.000296 & 0.0026 & 0.228 & 0.0000393 & $(-0.00000157,0.0000806)$ \\
\hline$C_{4,0}$ & 0.000288 & 0.0026 & 0.221 & 0.0000464 & $(0.00000740,0.0000871)$ \\
\hline
\end{tabular}


(a)

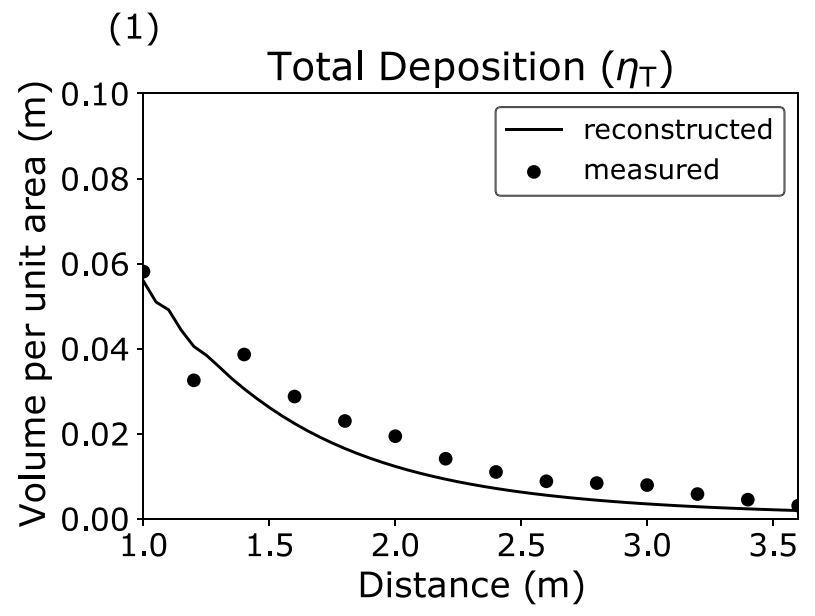

(b)

(1)

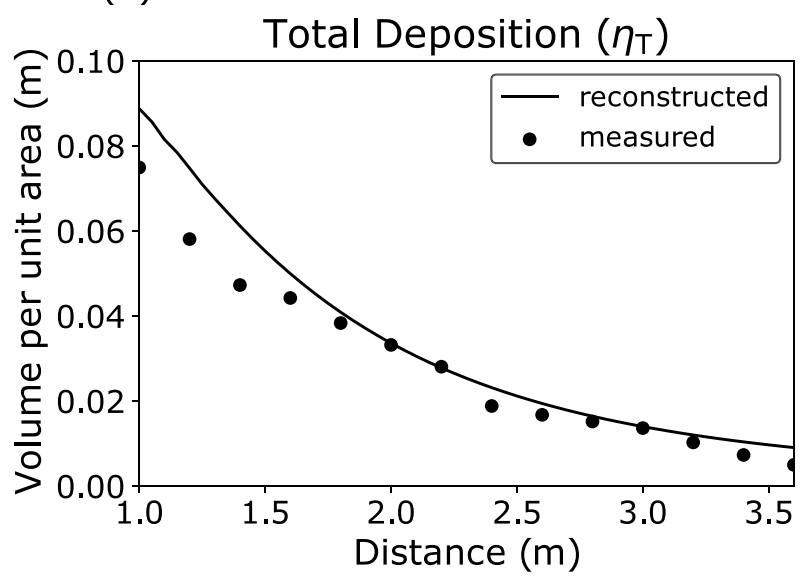

(2)

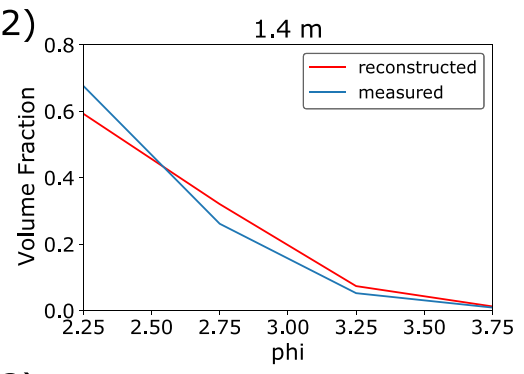

(3)

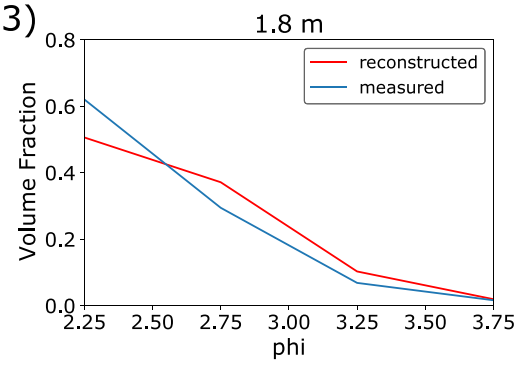

(2)

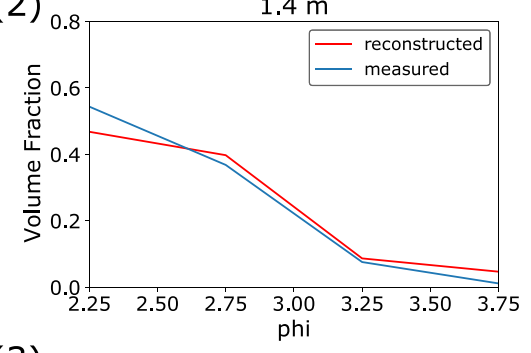

(3)

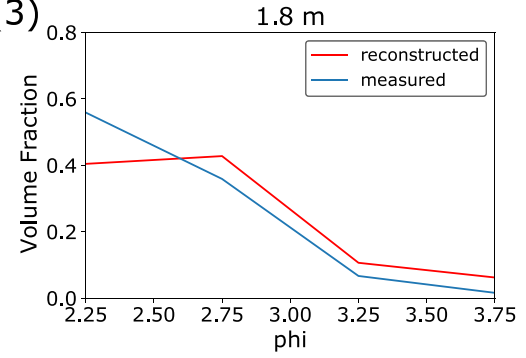

Figure 7. Reconstructed deposit profiles and sampled deposit data of experiments PP1 and PP2. (a) (1) Reconstructed and sampled $\eta_{\mathrm{T}}$ of PP1. (2) Grain size distribution at $1.4 \mathrm{~m}$ downstream. (3) Grain size distribution at $1.8 \mathrm{~m}$ downstream. (b) (1) Reconstructed and sampled $\eta_{\mathrm{T}}$ of PP2. (2) Grain size distribution at $1.4 \mathrm{~m}$ downstream. (3) Grain size distribution at $1.8 \mathrm{~m}$ downstream.

flow velocity $U_{x_{U}}$, the measured value of PP1 was $0.0812 \mathrm{~m} / \mathrm{s}$ and the reconstructed value was $0.793 \mathrm{~m} / \mathrm{s}$ with an uncertainty range of $\pm 0.00360 \mathrm{~m} / \mathrm{s}$. The measured $U_{x_{U}}$ of PP2 was $0.924 \mathrm{~m} / \mathrm{s}$ and the reconstructed value was $0.109 \mathrm{~m} / \mathrm{s}$ with an uncertainty range of $\pm 0.00817 \mathrm{~m} / \mathrm{s}$. The uncertainty range of reconstructed $U_{x_{U}}$ for both PP1 and PP2 were also relatively small in comparison to the measured and reconstructed values. The percent errors between reconstructed and measured $U_{x_{U}}$ were $2.38 \%$ and $17.9 \%$, of which that of PP1 was lower than that of PP2.

The measured value of flow duration $T_{\mathrm{d}}$ for PP1 was $936 \mathrm{~s}$ and the reconstructed value was $494 \mathrm{~s}$ with an uncertainty range of $\pm 58.6 \mathrm{~s}$. The measured $T_{\mathrm{d}}$ for PP2 was $966 \mathrm{~s}$ and the reconstructed value was $920 \mathrm{~s}$ with an uncertainty range of $\pm 72.6 \mathrm{~s}$. The percent errors between reconstructed and measured $T_{\mathrm{d}}$ were $47.2 \%$ (PP1) and $4.76 \%$ (PP2). Reconstructed values for $T_{\mathrm{d}}$ were lower than the measured values (Table 5). The measured value of total flow concentration $C_{\mathrm{T}, x_{C}}$ for PP1 was 0.000808 and the reconstructed value was 0.00702 with an uncertainty range of \pm 0.000667 . The measured $C_{\mathrm{T}, x_{C}}$ for PP2 was 0.00410 and the reconstructed value was 0.00344 with an uncertainty range of \pm 0.000462 . The percent errors between reconstructed and 
(a)

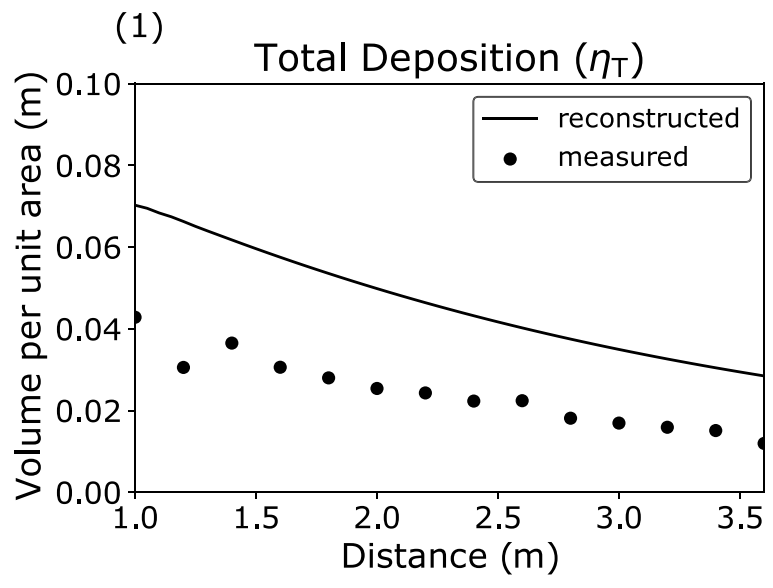

(b)

\section{(1)}

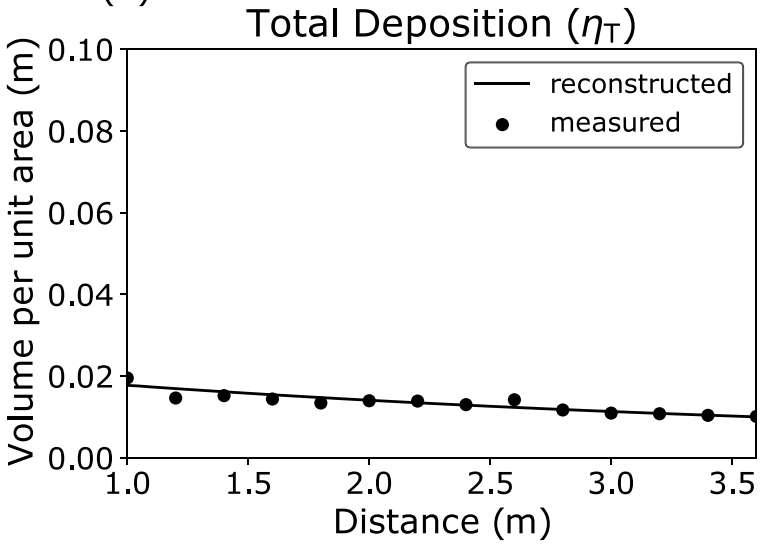

(c)

(1)

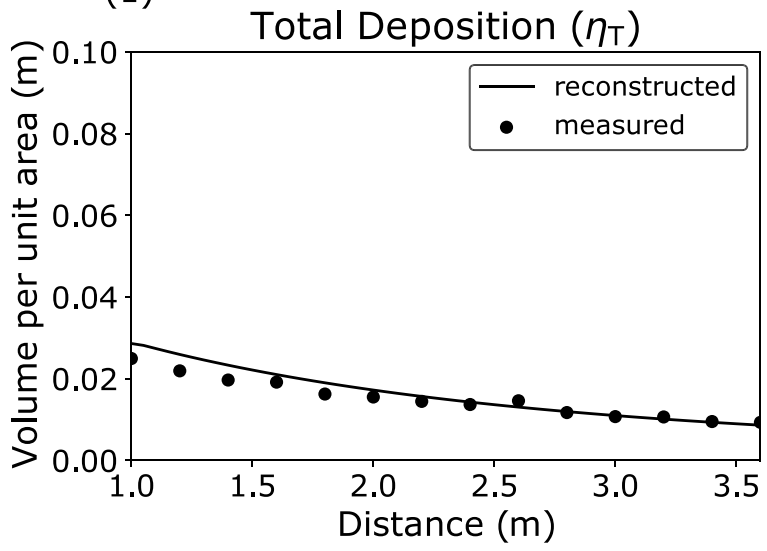

(2)
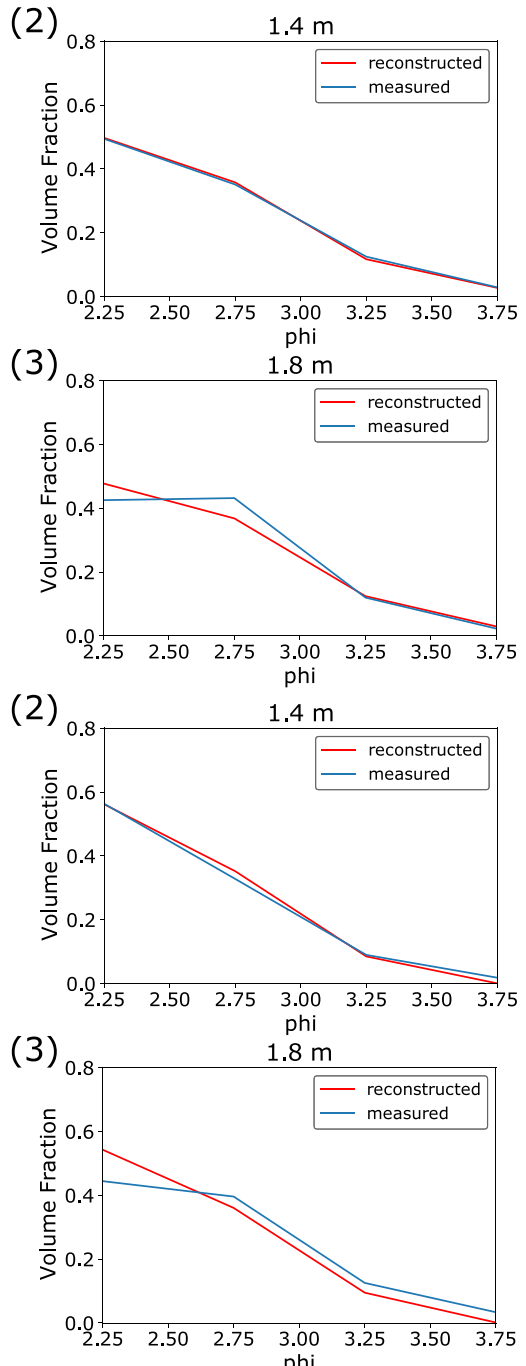

(2)
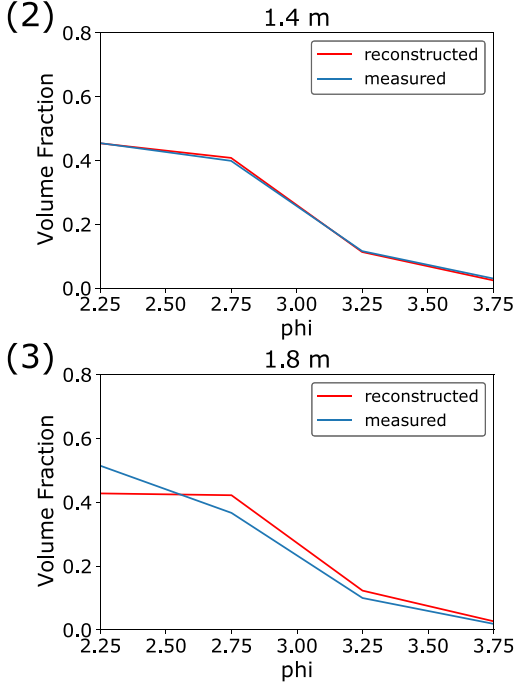

Figure 8. Reconstructed deposit profiles and the sampled deposit data of experiments PP3, PP4 and PP5. (a) (1) Reconstructed and sampled $\eta_{\mathrm{T}}$ of PP3. (2) Grain size distribution at $1.4 \mathrm{~m}$ downstream. (3) Grain size distribution at $1.8 \mathrm{~m}$ downstream. (b) (1) Reconstructed and sampled $\eta_{\mathrm{T}}$ of PP4. (2) Grain size distribution at $1.4 \mathrm{~m}$ downstream. (3) Grain size distribution at $1.8 \mathrm{~m}$ downstream. (c) (1) Reconstructed and sampled $\eta_{\mathrm{T}}$ of PP5. (2) Grain size distribution at $1.4 \mathrm{~m}$ downstream. (3) Grain size distribution at $1.8 \mathrm{~m}$ downstream. 
Table 5

Measured and Reconstructed Flow Conditions for Experiments PP1 and PP2

\begin{tabular}{|c|c|c|c|c|c|c|}
\hline Parameters & PP1 R. & PP1 M. & Percent Error & PP2 R. & PP2 M. & Percent Error \\
\hline$C_{\mathrm{T}, x_{C}}$ & $0.00702 \pm 0.000667$ & 0.000808 & $768 \%$ & $0.00344 \pm 0.000462$ & 0.00410 & $16.1 \%$ \\
\hline$C_{1, x_{C}}$ & $0.00333 \pm 0.000274$ & 0.0000911 & $3,560 \%$ & $0.000623 \pm 0.0000924$ & 0.000612 & $1.80 \%$ \\
\hline$C_{2, x_{C}}$ & $0.00250 \pm 0.000244$ & 0.000389 & $541 \%$ & $0.00135 \pm 0.000189$ & 0.00224 & $39.9 \%$ \\
\hline$C_{3, x_{C}}$ & $0.000915 \pm 0.000125$ & 0.000228 & $302 \%$ & $0.000683 \pm 0.0000834$ & 0.000944 & $27.7 \%$ \\
\hline$C_{4, x_{C}}$ & $0.000272 \pm 0.0000509$ & 0.0000999 & $172 \%$ & $0.000788 \pm 0.000106$ & 0.000303 & $160 \%$ \\
\hline$H_{x_{H}}(\mathrm{~m})$ & $0.157 \pm 0.00921$ & 0.116 & $35.1 \%$ & $0.142 \pm 0.00849$ & 0.123 & $15.3 \%$ \\
\hline$U_{x_{U}}(\mathrm{~m} / \mathrm{s})$ & $0.0793 \pm 0.00360$ & 0.0812 & $2.38 \%$ & $0.109 \pm 0.00817$ & 0.0924 & $17.9 \%$ \\
\hline$T_{\mathrm{d}}(\mathrm{s})$ & $494 \pm 58.6$ & 936 & $47.2 \%$ & $920 \pm 72.6$ & 966 & $4.76 \%$ \\
\hline
\end{tabular}

$\mathrm{M}$, measured; R, reconstructed.

measured $C_{\mathrm{T}, x_{C}}$ were $768 \%$ (PP1) and 16.1\% (PP2), of which that of PP1 had a significantly larger deviation than that of PP2. The reconstructed values of each grain-size class were mostly overestimated (Figure 9d).

\subsubsection{Experiments Conducted With $8.00 \%$ Slope (PP3, PP4, and PP5)}

Similar to the results of PP1 and PP2, deposit profiles in experiments PP3, PP4, and PP5 showed thinning and fining downstream trends. The reconstructed deposit profiles of the total deposition closely matched the sampled data for PP4 and PP5 (Figures 8b1 and 8c1) but was slightly greater than the measured values for PP3 (Figure 8a1). The reconstructed grain size distributions at $1.4 \mathrm{~m}$ and $1.8 \mathrm{~m}$ downstream agreed well with the measured values for all three experiments (Figures 8a2, 8a3, 8b2, 8b3, 8c2 and 8c3).

The measured $H_{x_{H}}$ of PP3 was $0.149 \mathrm{~m}$ and the reconstructed value was $0.192 \mathrm{~m}$ with an uncertainty range (95\% confidence interval) of $\pm 0.0145 \mathrm{~m}$. The measured $H_{x_{H}}$ of PP4 was $0.232 \mathrm{~m}$ and the reconstructed value was $0.258 \mathrm{~m}$ with an uncertainty range of $\pm 0.0180 \mathrm{~m}$. For PP5, the measured $H_{x_{H}}$ was $0.196 \mathrm{~m}$ and the reconstructed value was $0.126 \mathrm{~m}$ with an uncertainty range of $\pm 0.00925 \mathrm{~m}$. Reconstructed $H_{x_{H}}$ of PP3, PP4, and PP5 had a relatively small uncertainty range in comparison to the measured and reconstructed values. The percent errors between reconstructed and measured $H_{x_{H}}$ were $28.8 \%, 11.1 \%$, and $35.7 \%$ for PP3, PP4 and PP5, respectively (Table 6). Of these values, that of PP5 was slightly higher than those of PP3 and PP4. The measured $U_{x_{U}}$ of PP3 was $0.113 \mathrm{~m} / \mathrm{s}$ and the reconstructed value was $0.150 \mathrm{~m} / \mathrm{s}$ with an uncertainty

Table 6

Flow Conditions Measured and Reconstructed for Experiments PP3, PP4 and PP5

\begin{tabular}{|c|c|c|c|c|c|c|c|c|c|}
\hline Parameters & PP3 R. & PP3 M. & $\begin{array}{l}\text { Percent } \\
\text { Error }\end{array}$ & PP4 R. & PP4 M. & $\begin{array}{l}\text { Percent } \\
\text { Error }\end{array}$ & PP5 R. & PP5 M. & $\begin{array}{c}\text { Percent } \\
\text { Error }\end{array}$ \\
\hline$C_{\mathrm{T}, x_{C}}$ & $0.00580 \pm 0.000443$ & 0.00227 & $155 \%$ & $0.00151 \pm 0.000385$ & 0.00533 & $71.7 \%$ & $0.00564 \pm 0.000342$ & 0.00331 & $70.1 \%$ \\
\hline$C_{1, x_{C}}$ & $0.00142 \pm 0.000120$ & 0.000108 & $1,210 \%$ & $0.000379 \pm 0.000106$ & 0.000884 & $57.1 \%$ & $0.00108 \pm 0.0000556$ & 0.000258 & $321 \%$ \\
\hline$C_{2, x_{C}}$ & $0.00218 \pm 0.000167$ & 0.00136 & $59.9 \%$ & $0.000534 \pm 0.000126$ & 0.00313 & $82.9 \%$ & $0.00227 \pm 0.000116$ & 0.00210 & $7.80 \%$ \\
\hline$C_{3, x_{C}}$ & $0.00149 \pm 0.000104$ & 0.000646 & $1,310 \%$ & $0.000420 \pm 0.0000814$ & 0.00109 & $61.5 \%$ & $0.00149 \pm 0.000124$ & 0.000793 & $88.1 \%$ \\
\hline$C_{4, x_{C}}$ & $0.000713 \pm 0.000105$ & 0.000157 & $3,540 \%$ & $0.000175 \pm 0.0000716$ & 0.000227 & $22.9 \%$ & $0.000793 \pm 0.0000685$ & 0.000159 & $3,980 \%$ \\
\hline$H_{x_{H}}(\mathrm{~m})$ & $0.192 \pm 0.0145$ & 0.149 & $28.8 \%$ & $0.258 \pm 0.0180$ & 0.232 & $11.1 \%$ & $0.126 \pm 0.00925$ & 0.196 & $35.7 \%$ \\
\hline$U_{x_{U}}(\mathrm{~m} / \mathrm{s})$ & $0.150 \pm 0.00508$ & 0.113 & $33.2 \%$ & $0.172 \pm 0.00147$ & 0.109 & $57.6 \%$ & $0.183 \pm 0.00451$ & 0.137 & $73.7 \%$ \\
\hline$T_{\mathrm{d}}(\mathrm{s})$ & $687 \pm 82.5$ & 740 & $7.16 \%$ & $974 \pm 46.8$ & 332 & $193 \%$ & $264 \pm 17.4$ & 408 & $35.3 \%$ \\
\hline
\end{tabular}

$\mathrm{M}$, measured; R, reconstructed. 
(a)

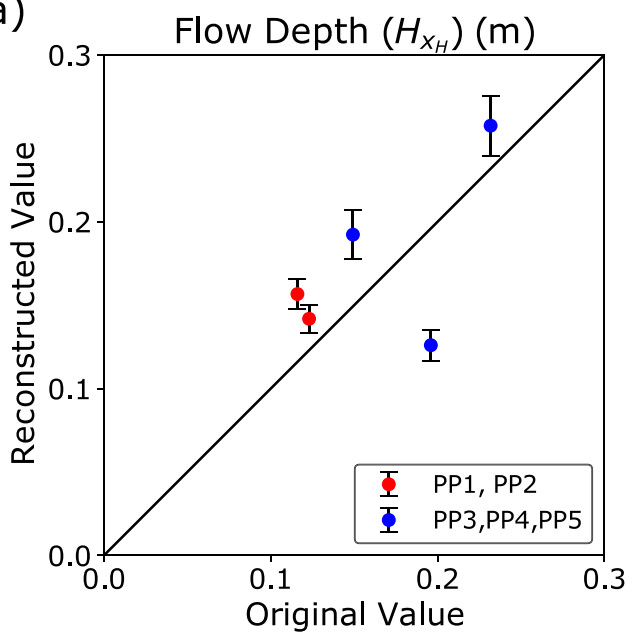

(c)

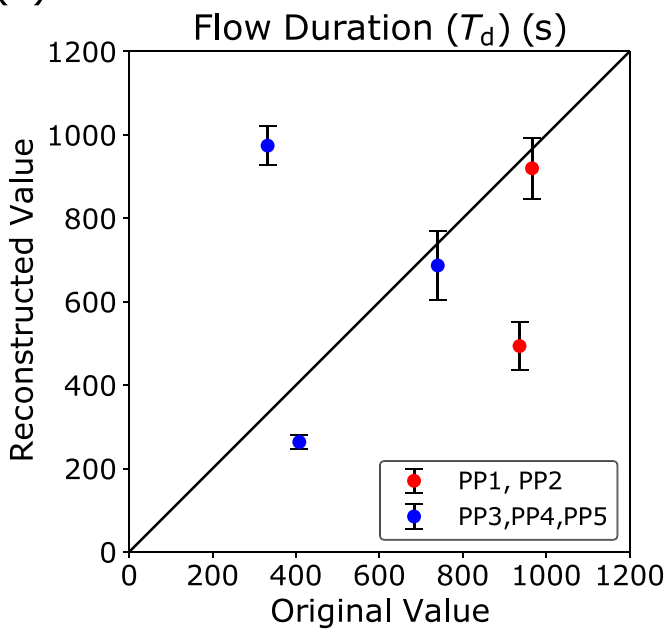

(b)

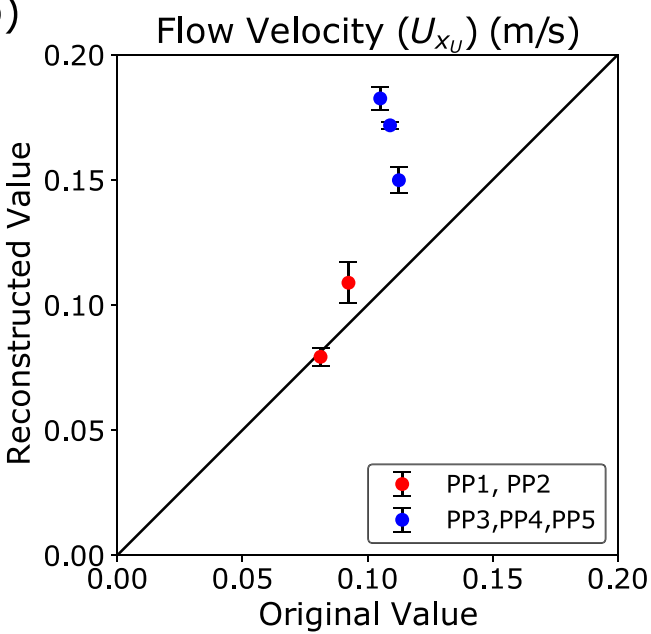

(d)

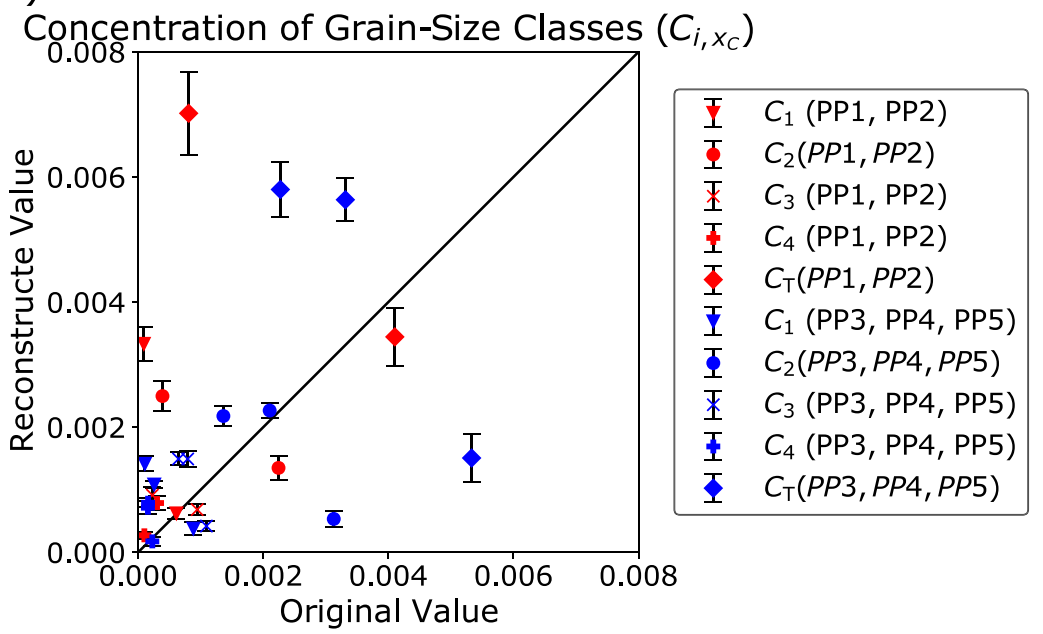

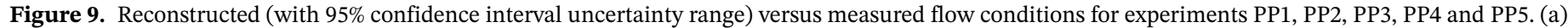
Plot for $H_{x_{H}}$. (b) Plot for $U_{x_{U}}$. (c) Plot for $T_{\mathrm{d}}$. (d) Plot for $C_{i, x_{C}}$.

range of $\pm 0.00508 \mathrm{~m} / \mathrm{s}$. The measured $U_{x_{U}}$ of PP4 was $0.109 \mathrm{~m} / \mathrm{s}$ and the reconstructed value was $0.172 \mathrm{~m} / \mathrm{s}$ with an uncertainty range of $\pm 0.00147 \mathrm{~m} / \mathrm{s}$. For PP5, the measured $U_{x_{U}}$ was $0.137 \mathrm{~m} / \mathrm{s}$ and the reconstructed value was $0.183 \mathrm{~m} / \mathrm{s}$ with an uncertainty range of $\pm 0.00451 \mathrm{~m} / \mathrm{s}$. Reconstructed $U_{x_{U}}$ of PP3, PP4 and PP5 had a relatively small uncertainty range in comparison to the measured and reconstructed values. The percent errors between reconstructed and measured $U_{x_{U}}$ were $33.2 \%$ (PP3), 57.6\% (PP4), and 73.7\% (PP5), in which PP5 also exhibited a deviation higher than those of PP3 and PP4 (Figure 9b).

The measured $T_{\mathrm{d}}$ of PP3 was $740 \mathrm{~s}$ and the reconstructed value was $689 \mathrm{~s}$ with an uncertainty range of \pm $82.5 \mathrm{~s}$. The measured $T_{\mathrm{d}}$ of PP4 was $332 \mathrm{~s}$ and the reconstructed value was $974 \mathrm{~s}$ with an uncertainty range of $\pm 46.8 \mathrm{~s}$. For PP5, the measured $T_{\mathrm{d}}$ was $408 \mathrm{~s}$ and the reconstructed value was $264 \mathrm{~s}$ with an uncertainty range of $\pm 17.4 \mathrm{~s}$. The percent errors between reconstructed and measured $T_{\mathrm{d}}$ were $7.16 \%$ (PP3), 193\% (PP4), and 35.3\% (PP5), of which PP4 showed a much larger deviation than PP3 and PP5. The measured $C_{\mathrm{T}, x_{C}}$ of PP3 was 0.00227 and the reconstructed value was 0.00580 with an uncertainty range of \pm 0.000443 . The measured $C_{\mathrm{T}, x_{C}}$ of PP4 was 0.00533 and the reconstructed value was 0.00151 with an uncertainty range of \pm 0.000385. For PP5, the measured $C_{\mathrm{T}, x_{C}}$ was 0.00331 and the reconstructed value was 0.00564 with an uncertainty range of \pm 0.000342 . The percent errors between reconstructed and measured $C_{\mathrm{T}, x_{C}}$ were $155 \%$ (PP3), 71.7\% (PP4), and 70.1\% (PP5), where PP3 showed a greater deviation than the other two experiments. The concentrations of individual grain-size classes were mostly overestimated (Figure 9d). 


\section{Discussion}

\subsection{Validation of DNN as an Inversion Method for Turbidity Currents Using Numerical Test Data Sets}

Verification results using numerical data sets proved the ability of the DNN model to reasonably reconstruct the hydraulic conditions of turbidity currents from turbidites. Reconstructions of initial flow conditions and the flow duration using numerical data sets (Sections 5.1.1 and 5.1.2) were good judging from the $s$ and $B$ values (Tables 3 and 4). The reconstructions of the flow duration $T_{\mathrm{d}}$, flow depth $H_{0}$, velocity $U_{0}$, and sediment concentrations $C_{1,0}, C_{2,0}, C_{3,0}$, and $C_{4,0}$ showed high (Tables 3 and 4).

Correlations between the actual and reconstructed values were observed for all parameters. Some outliers were observed for the reconstructed parameters, but most of the reconstructed values were close to the perfect reconstruction line. The range of misfit $(2 s)$ of all parameters was below $23.0 \%$ of the matching representative value (Tables 3 and 4). A relatively greater degree of scattering was observed for $T_{\mathrm{d}}$ compared to the other parameters (Figures 5 and $6 \mathrm{~b}$ ).

Concerning the estimation bias, zero was included in the $95 \%$ CI of bias for most of the parameters, proving that the reconstructed values were not significantly biased with respect to the original values. Even among parameters where statistically significant biases were detected, their deviations were minor compared with the representative values of the parameters (Tables 3 and 4). For example, in both numerical data sets of experiments conducted with $10.0 \%$ slope and $8.00 \%$ slope, the estimation bias $B$ for $T_{\mathrm{d}}$ had a negative value and the range of the CI of $B$ was below zero (Tables 3 and 4), indicating a tendency of underestimation for $T_{\mathrm{d}}$. However, the bias for $T_{\mathrm{d}}$ was only $7.84 \%$ (10.0\% slope numerical data sets) or $7.51 \%$ (8.00\% slope numerical data sets) of the representative value of this parameter $(630 \mathrm{~s})$.

Thus, this method is suitable for estimating the paleo-hydraulic conditions of actual experimental scale turbidity currents. Correlation between reconstructed parameters and original values did not show any significant bias, implying that the inverse model developed in this study served as a high precision, high accuracy estimator of flow conditions.

\subsection{Verification of DNN Inversion With Flume Experiment Data}

As a result of inversion using the DNN model, the overall deposit profiles were reasonably reconstructed for all five experiments, and the reconstructed grain size distribution downstream matched the sampled values from experiment deposits (Figures 7 and 8). The DNN model as an inverse method tries to find the combination of hydraulic conditions that best produces the inputted deposit profiles. The fact that the reconstructed hydraulic conditions reproduced the deposit profiles used for inverse analysis indicated the DNN inverse model performed well.

For the hydraulic conditions and flow duration, a good match was observed for $H_{x_{H}}$ for all five experiments with a percent error under $36.0 \%$ (Tables 5 and 6). Flow duration $T_{\mathrm{d}}$ was reasonably reconstructed for PP1, PP2, PP3, and PP5, with a percent error lower than $48.0 \%$. Reconstructed $T_{\mathrm{d}}$ of PP4 had a percent error greater than $190 \%$. The reconstructed concentrations of each grain-size class $C_{i, x_{C}}$ were mostly overestimated (Figure 9). The measured and reconstructed values of flow velocities $U_{x_{U}}$ agreed well, especially for PP1 and PP2, with a percent error less than $18.0 \% . U_{x_{U}}$ reconstructed for PP3, PP4, and PP5 ranged from $33.2 \%$ to $73.7 \%$.

The ability of the DNN model to distinguish minor differences in the characteristics of deposits was proved in the tests using numerical data sets, where a wide variety of initial conditions of flows were well reconstructed (Section 5.1). The fact that the reconstructed initial flow conditions by the DNN model for the 2,000 artificial test data sets lay very close to the line of perfect reconstruction showed that nonuniqueness of deposit was unlikely a problem for the range of flow conditions tested in this study. According to the analysis of the results of the application of the DNN model to flume experiment data, there are three sources of deviations in the reconstruction of hydraulic conditions: (a) measurement errors during and after the experiments, (b) bias inherent in the inverse model, and (c) inaccuracy within the forward model of turbidity currents. 
1. The main source of deviation for sediment concentrations $C_{i, x_{C}}$ may be inaccuracies in measurements. As shown in Figure 9, some of the measured concentrations $C_{i, x_{C}}$ were extremely small $(<0.1 \%)$, making them susceptible to minor disturbances during sampling and measurements. For extremely small values, even minor deviations appear to be large. Thus, for $C_{i, x_{C}}$, the main source of deviation may not be the reconstructed values but the measured values.

As for flow velocity $U_{x_{U}}$, the accuracy of measurement was greatly affected by the SNR during the experiments. Experiments PP3, PP4, and PP5 had relatively lower SNRs and a narrower range of reliable measurement than PP1 and PP2, with PP4 and PP5 having the lowest SNR (Appendix A). The narrower range of reliable measurement for PP3, PP4, and PP5 resulted in ranges of vertical velocity profile without measurements. The measured values closest to the velocity maximum was used for calculation for PP3, PP4, and PP5, which could be slightly smaller than the actual value. In which case, the calculated layer-averaged flow velocity would also be smaller than the actual value. This may be the reason that $U_{x_{U}}$ of experiments PP3, PP4 and PP5 were overestimated and showed larger deviation than PP1 and PP2.

Slight deviation in the sampling and measurement of the deposits could also be a source of deviation in the eventual reconstruction. The uncertainty range for the reconstructed parameters was calculated using Jackknife samples of the $\eta$ values measured from the experiment deposits. The width of the uncertainty range showed that slight deviation of the input $\eta$ values can propagate to the output reconstructed values of $H_{x_{U}}, U_{x_{U}}, C_{i, x_{C}}$, and $T_{\mathrm{d}}$.

2. Regarding the inherent bias in the inverse model, the reconstructed $T_{\mathrm{d}}$ for the experiments PP1, PP2, PP3, and PP5 exhibited the same tendencies of deviation during the reconstruction using numerical test data sets. Thus, deviation in the reconstruction of $T_{\mathrm{d}}$ may be partially due to systematic error originating from the internal settings of the DNN.

3. Inaccuracy in the forward model in describing the physical processes of turbidity currents may account for deviations of the reconstructed flow velocities from the measured values. There are several possible reasons why the reconstruction of flow velocity was not as accurate as with the other parameters, but the most probable reason is the inaccuracy of the entrainment function in describing the actual effect of entrainment in flow, considering that the exponent in the calculation of the dimensionless vertical velocity in the entrainment function was determined purely via optimization and differed greatly in previous studies (Dorrell et al., 2018; Garcia \& Parker, 1991; Parker et al., 1987). Another problem may lie in the layer averaging of flow velocity. Dorrell et al. (2014) had pointed out that vertical stratification of flow velocity and density fields reduces depth averaged hydrostatic pressure and enhances suspended sediment and momentum flux, proving that incorporating the effect of flow stratification can be essential for calculating turbidity currents. This research aims to verify the DNN model as a method of inverse analysis of turbidity currents. The improvement of the forward model, including entrainment function and velocity calculation, should be the next step in the inverse analysis study of turbidity currents.

A limitation of the inverse analysis is that it can only be conducted for flow that is depositional. Inverse analysis reconstructs the flow conditions from turbidite deposited by turbidity current, so the model would be unable to detect a non-depositional condition if it happened during a flow. Although unlikely in the current lab setting, there is a possibility that flow parameters cannot be reconstructed when different combinations of initial conditions produce the same deposit profile, which will be a problem to be resolved in the future when using field data. Compared to the analytical models, the shallow water model implemented provides some details of the internal structure of the flow, but also holds certain limitations due to its simplified calculation of flow dynamics. Nonetheless, the simplifications enable large batches of natural scale simulations to be performed. Overall, even though a certain amount of deviation was observed for all parameters, they mostly lie within a reasonable range and provided valuable insights into the development of flow and deposits over time.

\subsection{Comparison of DNN With Existing Methodologies}

Compared to previous methods of inverse analysis of turbidity currents, the inversion method using the DNN model has great advantages in terms of calculation cost and reconstruction accuracy. Previous inver- 
sion methods of turbidity currents seek to optimize flow initial conditions to a particular set of data collected from turbidites, which is extremely time-consuming for application to one data set and does not guarantee the general applicability of the methods to turbidite deposits (Lesshafft et al., 2011; Nakao et al., 2020; Parkinson et al., 2017). For example, a genetic algorithm used in Nakao et al. (2020) first initializes a population of parameters and then optimizes the population of parameters through selection and mutation. Eventually, the remaining parameters can successfully reconstruct target turbidite. However, each epoch of optimization requires the selection results from the previous epoch, and thus, the calculation of the forward model cannot be parallelized over epochs. In the adjoint method used by Parkinson et al. (2017), control variables within the forward model of turbidity currents are first initialized and inputted into the numerical model. The turbidite deposit profile is calculated and compared with the target values using a cost function. Gradients of the cost function (objective function) for control variables are calculated analytically. If the result is not optimal, the adjoint model will run, and control variables will be adjusted by descent method. The adjusted control variables will be re-inputted into the numerical model. This cycle is repeated until the reconstructed deposit profile is judged to be optimal. Thus, the iteration of calculation cannot be performed simultaneously. In contrast, the DNN model explores the general functional relationship between turbidite deposited and flow, allowing its applicability to turbidity currents in general. The forward model calculation to generate training data sets can be perfectly parallelized, thereby significantly reducing the amount of calculation time.

Since the parallelization of the forward model calculation significantly reduced the calculation time, a more accurate and realistic forward model with a heavier calculation load could be implemented. As a result, the forward model used in this research is much better at capturing the spatio-temporal evolution of turbidity current than the forward model used in previous research (Falcini et al., 2009; Parkinson et al., 2017). Falcini et al. (2009) used a steady flow forward model, whereas our forward model is a nonsteady flow model that reproduces the evolution of flow over time. The method implemented in Parkinson et al. (2017) omitted the effect of entrainment, which is a significant part of sediment transport in turbidity currents. As a result, their reconstructed values of flow depth, concentration, and grain diameter of the turbidite were $2.56 \mathrm{~km}$, $0.0494 \%$, and $103 \mu \mathrm{m}$, respectively (Parkinson et al., 2017). Compared to the objective values collected from the turbidite deposits, these values showed great deviations. In contrast, our predictions closely agreed with the original values and the effect of sediment suspension was incorporated in our forward model. Another improvement from previous research is that the forward model used in this study applies to turbidite data sets of multiple grain-size classes.

Lesshafft et al. (2011) proposed a method based on direct numerical simulation (DNS) of the Navier-Stokes Equations. However, the calculation costs of the method were extremely high, making it impractical to apply the method to natural scale turbidites. The computational cost of DNS was scaled to $\mathrm{Re}^{3}$, thereby limiting the effectiveness of DNS to only experimental scale flows (Biegert et al., 2017). As a result, the maximum value of Reynolds number attained in previous numerical simulation using DNS was 15,000 (Cantero et al., 2007), which corresponds to $3.0 \mathrm{~cm} / \mathrm{s}$ for flow velocity and $50 \mathrm{~cm}$ for the flow depth. Thus, their methodology cannot be applied to natural scale turbidites.

\section{Conclusions}

In this study, a new method for the inverse analysis of turbidites using a DNN model was verified with actual flume experiment data. Compared to previous methods, the DNN model proved to be an efficient method for the inverse analysis of turbidity currents without compromising reconstruction accuracy. The DNN model performed well for verification using numerical data sets, judging by the standard deviation and bias of the reconstructed parameters. In terms of the application of the DNN model to experiment data, deposit profiles were well reconstructed; however, the initial flow conditions did not match the measured values perfectly. The uncertainty range of $95 \%$ confidence interval was determined for the reconstructed values of the experiment data sets using Jackknife resampling method.

The reconstructed and measured flow depths $H$ had percent error that is, less than $36.0 \%$, which is low for the inverse analysis results. The inverse analysis result for flow duration $T_{\mathrm{d}}$ had a percent error ranging from $4.76 \%$ to $35.2 \%$, except for PP4, which had a percent error of $193 \%$. Flow velocity $U$ was well reconstructed 
for experiments PP1 and PP2 (percent error 2.38\% and 17.9\%) and showed greater deviation for PP3, PP4, and PP5 (percent error 33.2\%-73.7\%). The reconstructed values for flow concentration of the $i$ th grain-size class $C_{i}$ had percent errors ranging from $1.79 \%$ to greater than $300 \%$.

Overall, the DNN model exhibited a good performance for the inversion of numerical data sets and some parameters of the experiment data. The deposit profiles were well reconstructed, demonstrating the success of the DNN model in exploring the functional relationship between the initial conditions of flow and resulting deposits. The verification results with numerical data sets and flume experiments reveal that the implemented forward model is competent in performing inverse analysis on turbidity currents, but it needs to be more robust for application to a wide range of flow conditions. Improvement of the forward models and parameters, such as the entrainment function, will be a top priority in the future. The DNN's hyperparameter settings and internal structure also have room for improvement, judging from the inversion result using numerical data sets. The application of the DNN model to field data sets will be the eventual goal.

\section{Appendix A: Flow Velocity Profile and the Corresponding Signal-to-Noise Ratio (SNR)}

The accuracy of flow velocity measurements by the acoustic Doppler velocity profiler (ADVP) used (Nortek Vectrino Profiler) was affected by the singal-to-noise ratio (SNR). According to the user manual of Nortek Vectrino Profiler, the "weak spot" of acoustic profile measurement due to pulse interference can be detected from the SNR values. The manual states that the SNR value of measurements need to be at least $30 \mathrm{~dB}$ to be considered reliable. Data with SNR between 20 and $30 \mathrm{~dB}$ should be used with caution and data with SNR lower than $20 \mathrm{~dB}$ should not be trusted. The measured velocity profile for each experiment and the matching SNR profile are shown in Figures A1 and A2. The height above bed of ADVP differed for the experiments conducted, thus the range of measured profiles above bed were also different.

From Figures A1 and A2, it was apparent that SNR of velocity measurements for experiments PP1 and PP2 were much higher than those of experiments PP3, PP4, and PP5\enleadertwodots The SNR values of PP1 and PP2 were above $40 \mathrm{~dB}$. Experiment PP3 had slightly better SNR profile than PP4 and PP5, with the peak SNR above $40 \mathrm{~dB}$, but the lowest SNR barely above $30 \mathrm{~dB}$. Experiment PP4 had especially low SNR, with the peak SNR slightly above $30 \mathrm{~dB}$. SNR of PP5 was above $40 \mathrm{~dB}$ toward the bottom, but decreased below $30 \mathrm{~dB}$ toward the top. While the entire velocity profile can be used for analysis of PP1 and PP2, only regions with high SNR can be used for PP3, PP4, and PP5. 
(a)

(1)

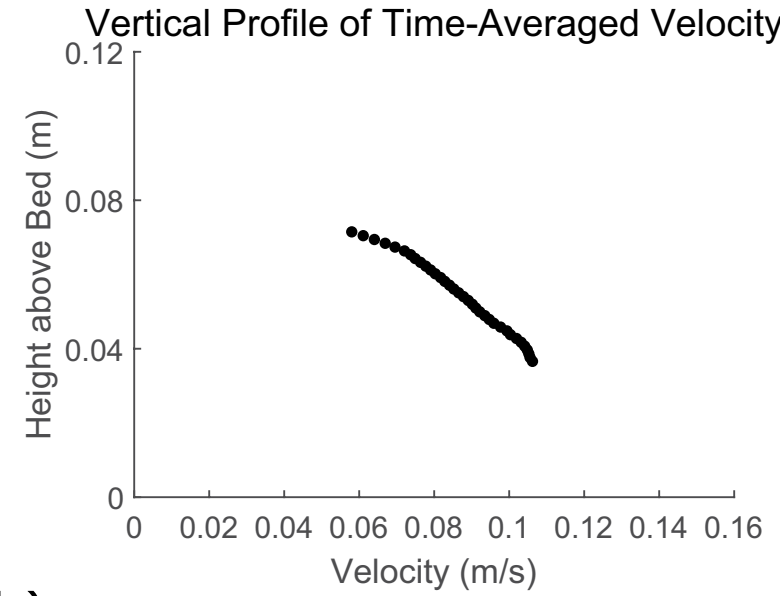

(b)

(1)

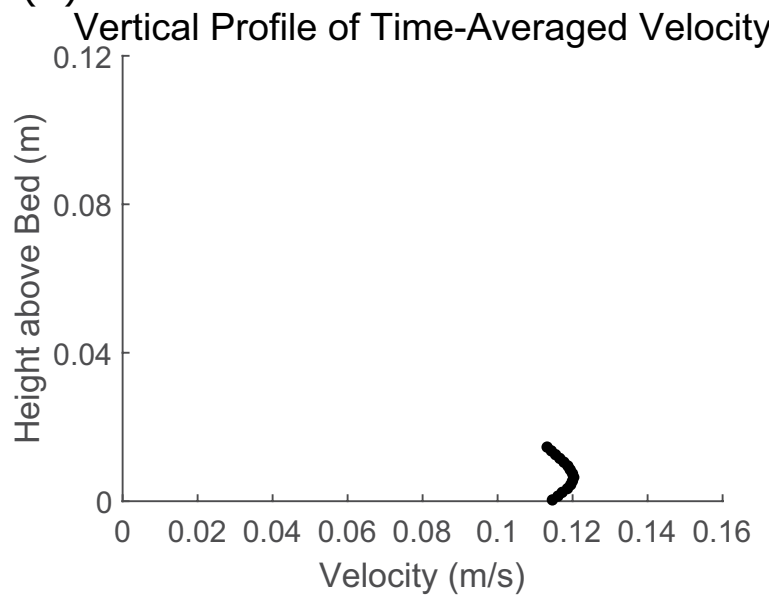

(2)

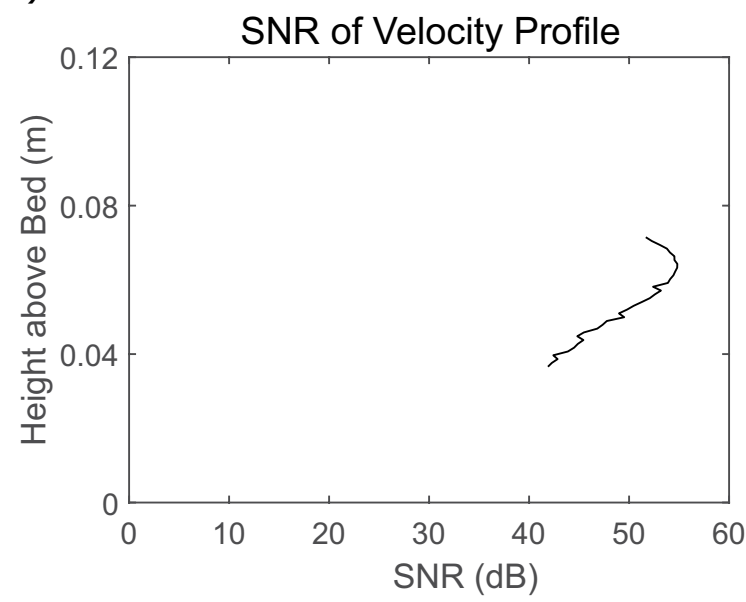

(2)

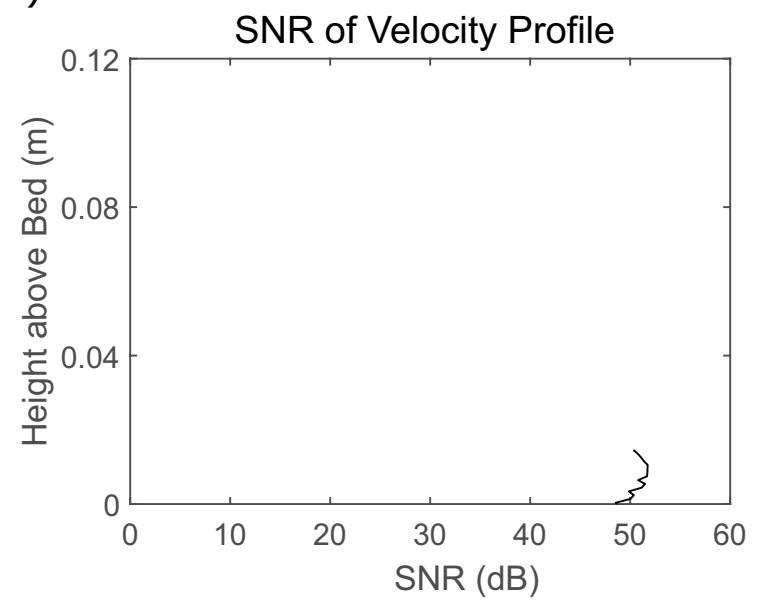

Figure A1. Time-averaged velocity profile and signal-to-noise ratio (SNR) of velocity profile for experiments conducted with $10.0 \%$ slope. (a) (1) Time-averaged velocity profile of PP1. (2) SNR of velocity profile for PP1. (b) (1) Time-averaged velocity profile of PP2. (2) SNR of velocity profile for PP2. 
(a)

(1)

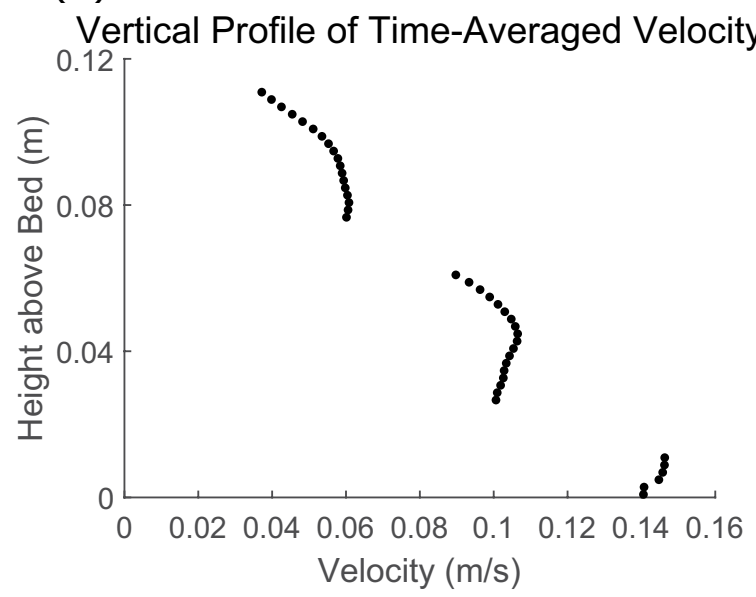

(b)

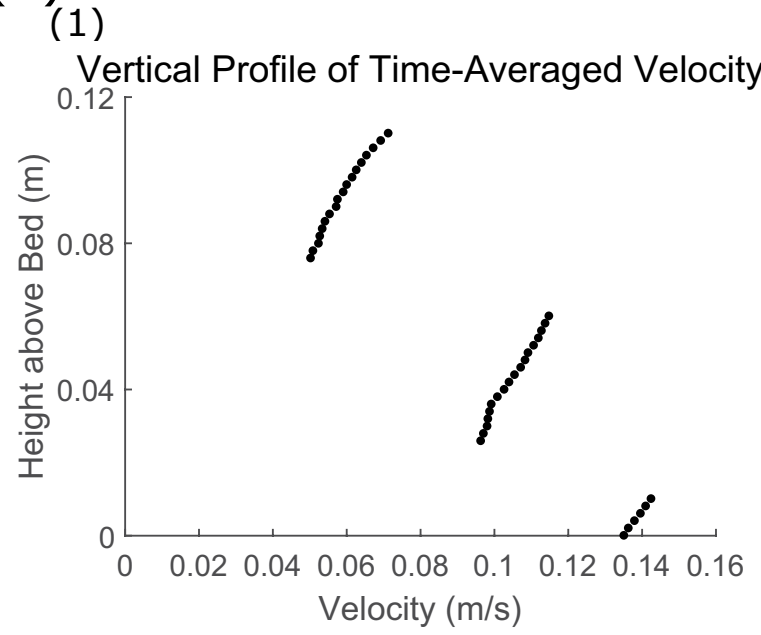

(c)

(1)

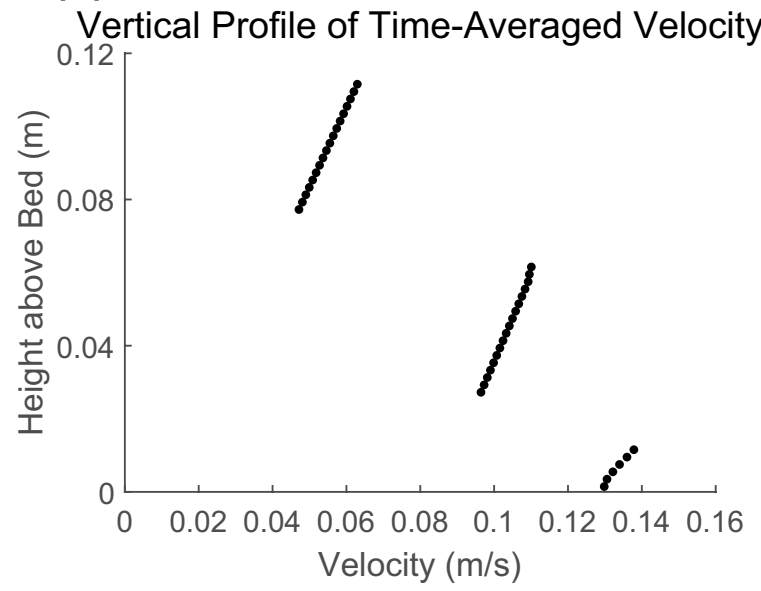

(2)

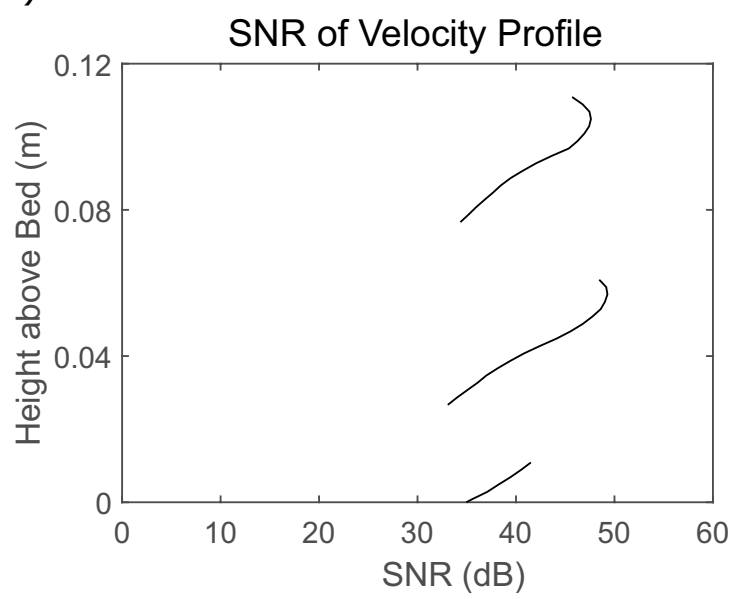

(2)

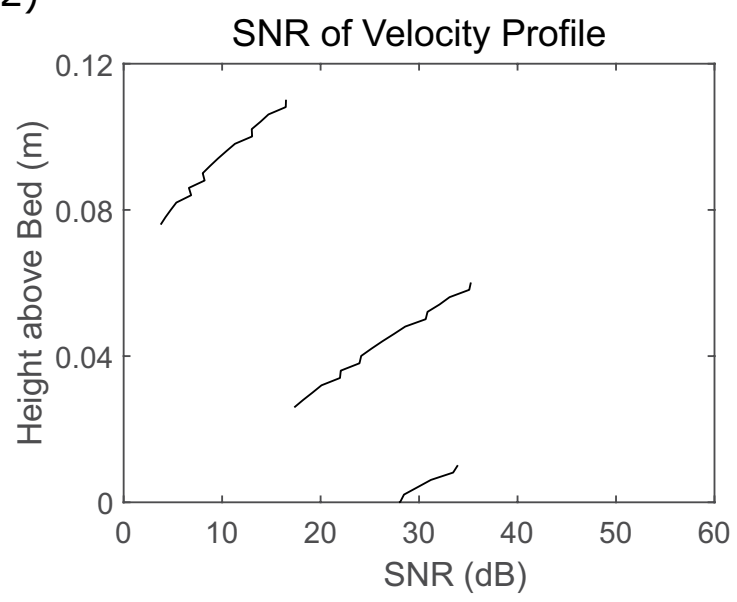

(2)

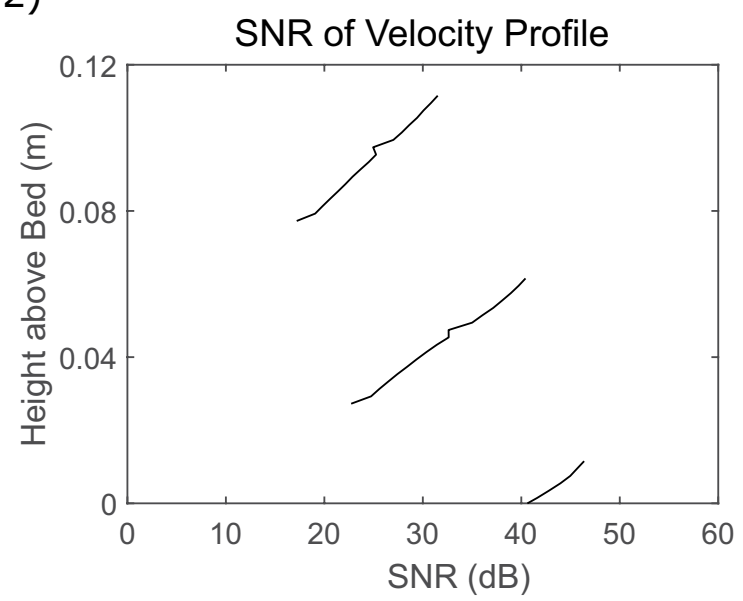

Figure A2. Time-averaged velocity profile and signal-to-noise ratio (SNR) of velocity profile for experiments conducted with $8.00 \%$ slope. (a) (1) Time-averaged velocity profile of PP3. (2) SNR of velocity profile for PP3. (b) (1) Time-averaged velocity profile of PP4. (2) SNR of velocity profile for PP4. (c) (1) Time-averaged velocity profile of PP5. (2) SNR of velocity profile for PP5. 


\section{Appendix B: Details of Forward Model Implemented}

\section{B1. Example of Forward Model Calculation}

The forward model was tested with two sets of numerical simulations of turbidity currents. Testing was conducted using the forward model programmed for generating numerical data sets for experiments conducted with a $10 \%$ slope. The settings of the numerical simulations are listed in Table B1, whereas the time evolution of the high $C_{\mathrm{T}, 0}, U_{0}$ simulation is shown in Figure B1, and the time evolution of the low $C_{\mathrm{T}, 0}$, $U_{0}$ simulation is shown in Figure B2. In both cases, the flow depth $H$ was greater toward the head of the current. $H$ at the head of the current also increased over time (Figures B1a and B2a). Flow velocity $U$ in the high $C_{\mathrm{T}, 0}, U_{0}$ simulation increased toward the head of the current (Figure B1b), whereas $U$ in the low $C_{\mathrm{T}, 0}, U_{0}$ simulation increased initially, and then decreased toward the head of the current (Figure B2b). The total volumetric concentration of sediment $C_{\mathrm{T}}$ in flow decreased downstream in both cases (Figures B1c and B2c). In the high $C_{\mathrm{T}, 0}, U_{0}$ case, a larger portion of sediment was deposited downstream than in the low $C_{\mathrm{T}, 0}, U_{0}$ case (Figures B1d and B2d). The low $C_{\mathrm{T}, 0}, U_{0}$ case had the most sediment deposited toward the upstream end of the flow.

Table B1

Initial Flow Conditions of Numerical Simulations of Turbidity Currents

\begin{tabular}{lcc}
\hline & High $C_{\mathrm{T}, 0}, U_{0}$ & Low $C_{\mathrm{T}, 0}, U_{0}$ \\
\hline$H_{0}(\mathrm{~m})$ & 0.15 & 0.15 \\
$U_{0}(\mathrm{~m} / \mathrm{s})$ & 0.2 & 0.02 \\
$C_{\mathrm{T}, 0}$ & 0.018 & 0.001 \\
$C_{1,0}$ & 0.004 & 0.0002 \\
$C_{2,0}$ & 0.005 & 0.0003 \\
$C_{3,0}$ & 0.0047 & 0.00027 \\
$C_{4,0}$ & 0.0043 & 0.00023 \\
$c_{\mathrm{f}}$ & 0.004 & 0.004 \\
$r_{\mathrm{o}}$ & 1.5 & 1.5 \\
Duration $(\mathrm{s})$ & 420 & 420 \\
\hline
\end{tabular}

For the both high and low $C_{\mathrm{T}, 0}, U_{0}$ simulations, a thicker deposit was observed for grain-size classes 1 and 2 than for grain-size classes 3 and 4 (Figures B1e, B1g, B1h, B2e, B2g and B2h). Although the initial concentrations of the finer grain-size classes 3 and $4 C_{3,0}, C_{4,0}$ were higher than that of the coarser grain-size class 1 $\left(C_{1,0}\right)$, less fine sediment was deposited since it was more likely to remain suspended and be carried beyond the lower flow boundary by the high-velocity flow. For the low $C_{\mathrm{T}, 0}, U_{0}$ simulation, the coarser grain-size class, grain-size classes 1 and 2, had almost all sediment deposited near the upstream boundary, whereas the finer grain-size class, grain-size classes 3 and 4, had sediment spread out toward the downstream direction (Figures B2e, B2f, B2g and B2h). This happened because the low-velocity flow was unable to keep the coarse sediment suspended.

\section{B2. Sensitivity Tests of Forward Model}

The degree of sensitivity of the forward model to changes in the initial conditions of the flow and model parameters was tested (Table B2). Testing was conducted using the forward model programmed for generating numerical data sets of experiments conducted with the $10 \%$ slope. Numerical simulations were conducted with different values of the six parameters $H_{0}, U_{0}, C_{\mathrm{T}, 0}, e_{\mathrm{s}}, r_{\mathrm{o}}$, and $c_{\mathrm{f}} . H_{0}, U_{0}$ and $C_{\mathrm{T}, 0}$ values in Case 1 were the mid-values over the range of $H_{0}, U_{0}$, and $C_{\mathrm{T}, 0}$ for generating training data. Other parameters remained constant for the simulations. 
(a)

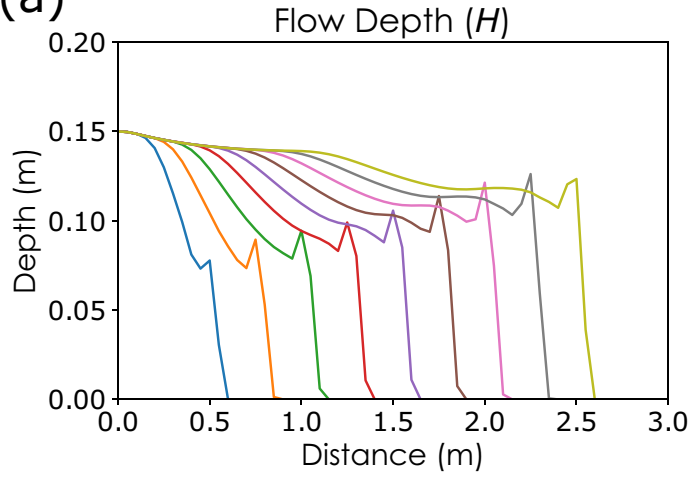

(c)

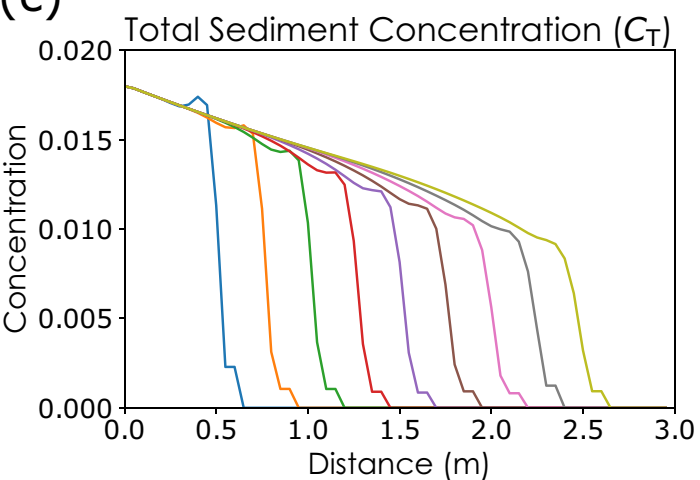

(e)

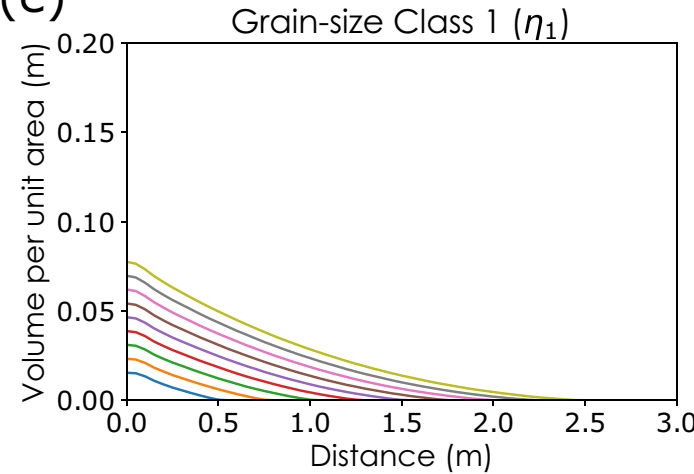

(

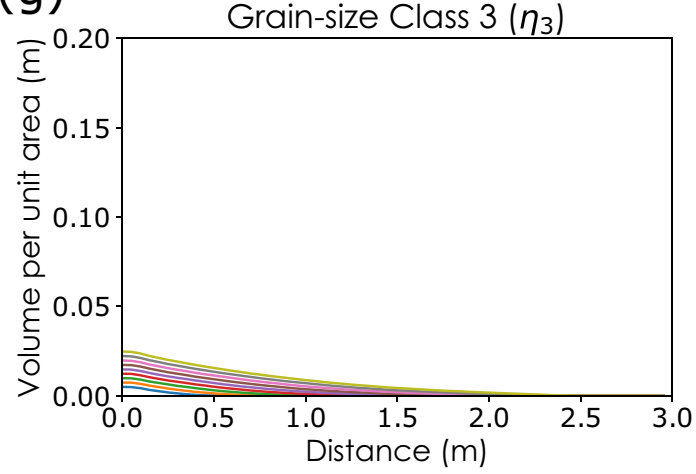

(b)

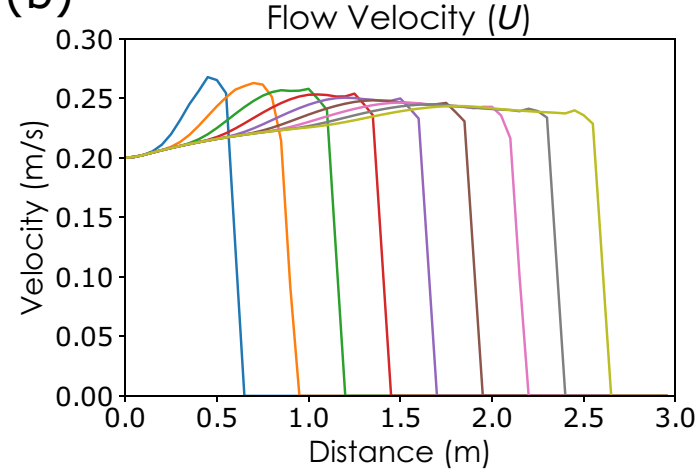

(d)

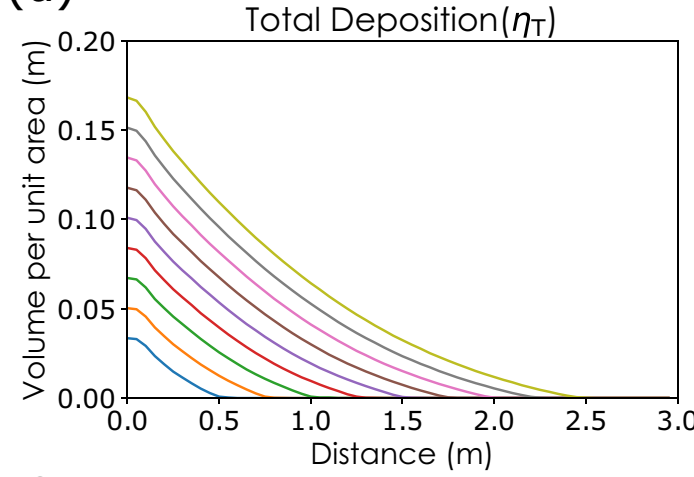

(f)

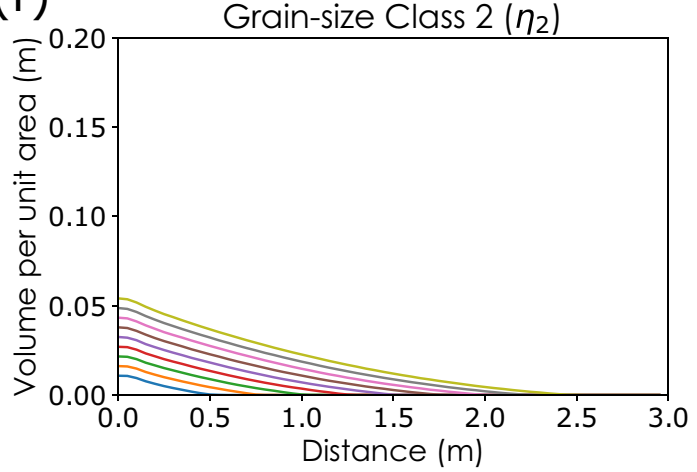

(h)

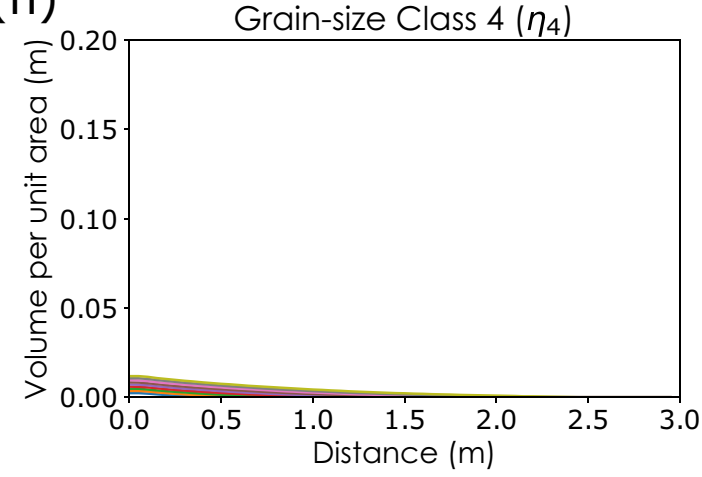

Figure B1. Example of forward model calculation with high initial flow velocity and sediment concentration (Table B1). (a) Time evolution of flow depth $H$. (b) Time evolution of flow velocity $U$. (c) Time evolution of total sediment volumetric concentration $C_{\mathrm{T}}$. (d) Time evolution of deposit profile $\eta_{\mathrm{T}}$. (e) Time evolution deposit profile of grain-size class $1 \eta_{1}$. (f) Time evolution of deposit profile of grain-size class $2 \eta_{1}$. (g) Time evolution of deposit profile of grain-size class $3 \eta_{1}$. (h) Time evolution of deposit profile of grain-size class $4 \eta_{1}$. 
(a)

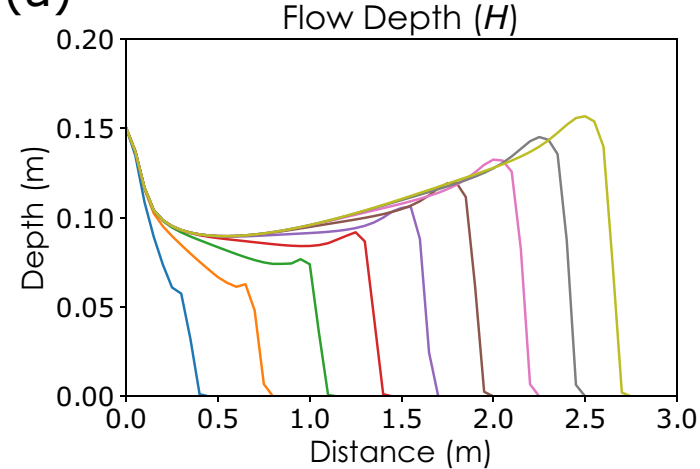

(c)

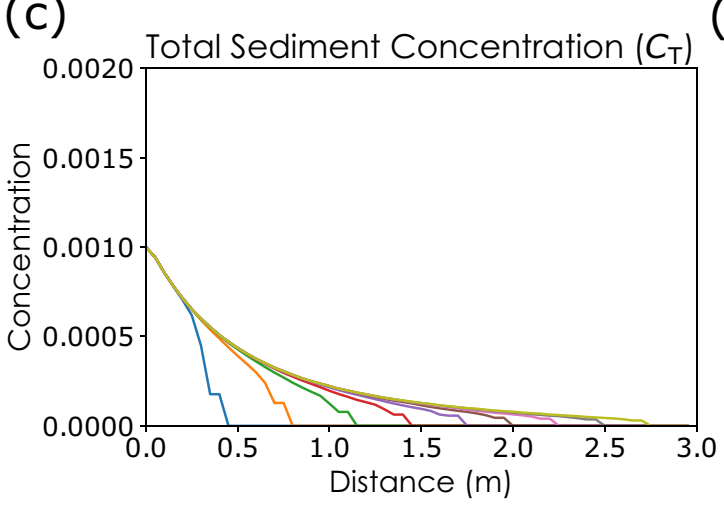

(e)

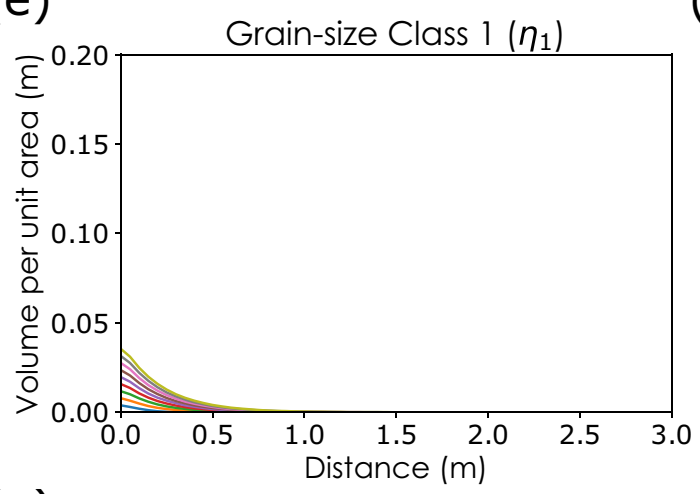

(

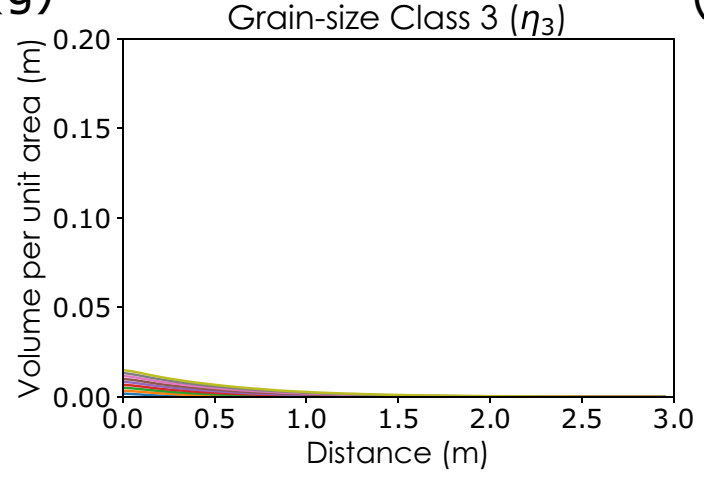

(b)

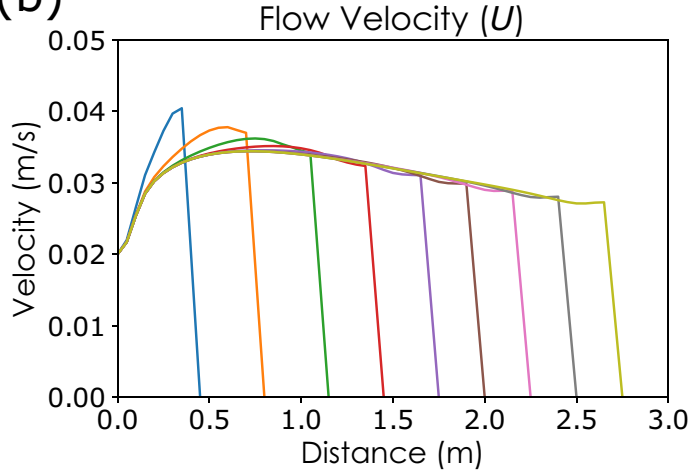

(d)

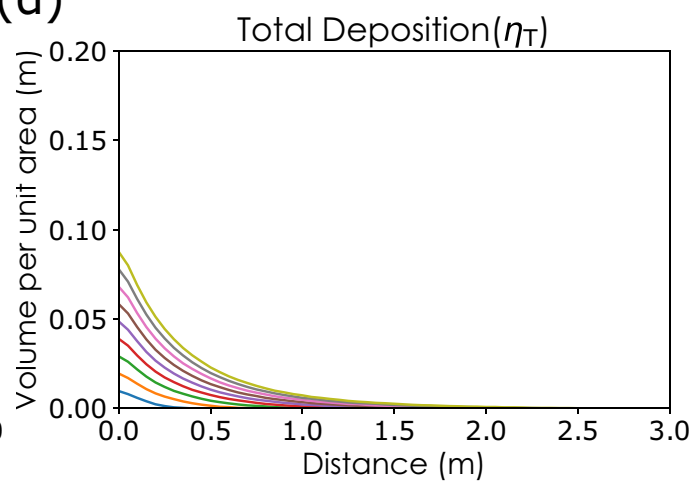

(f)

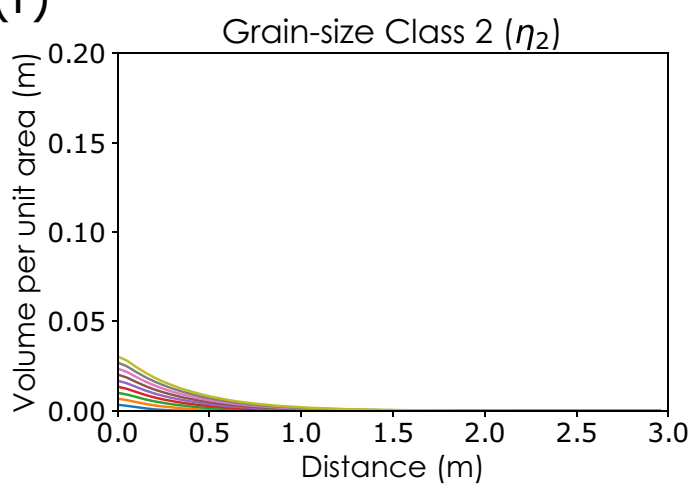

(h)

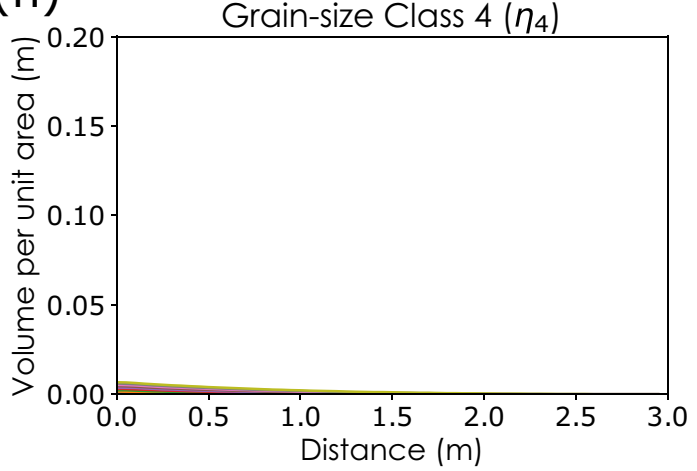

Figure B2. Example of forward model calculation with low initial flow velocity and sediment concentration (Table B1). (a) Time evolution of flow depth $H$. (b) Time evolution of flow velocity $U$. (c) Time evolution of total sediment volumetric concentration $C_{\mathrm{T}}$. (d) Time evolution of deposit profile $\eta_{\mathrm{T}}$. (e) Time evolution deposit profile of grain-size class $1 \eta_{1}$. (f) Time evolution of deposit profile of grain-size class $2 \eta_{1}$. (g) Time evolution of deposit profile of grain-size class $3 \eta_{1}$. (h) Time evolution of deposit profile of grain-size class $4 \eta_{1}$. 
(a)

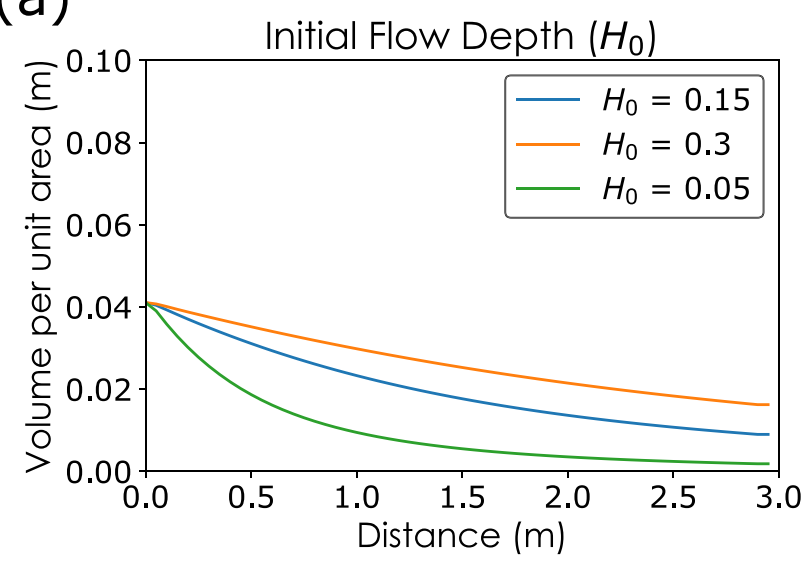

(c)

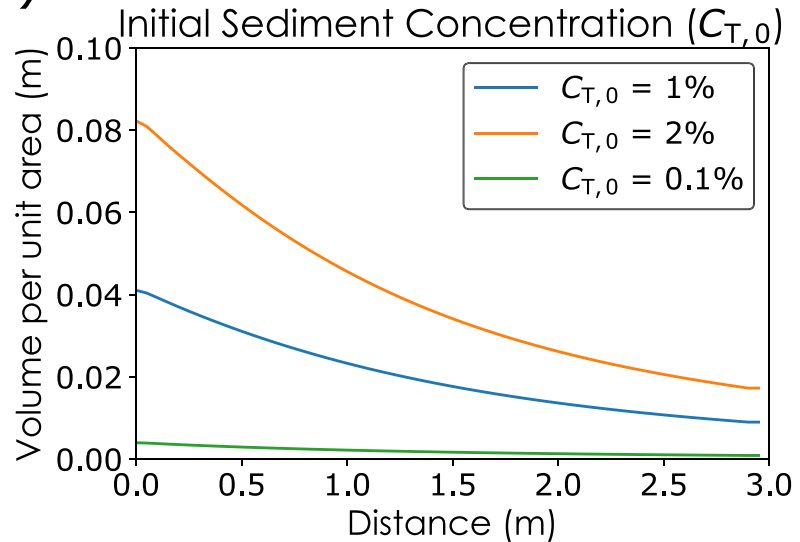

(e)

Ratio of Near-bed to Layer-averaged

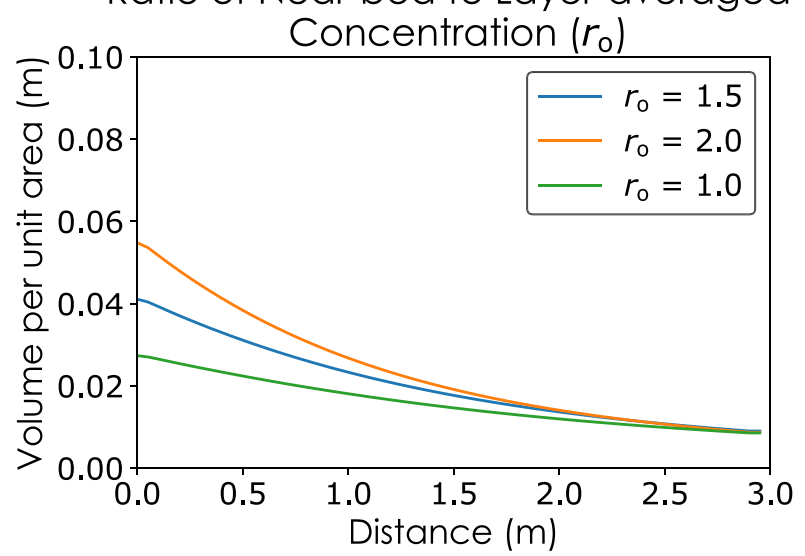

(b)

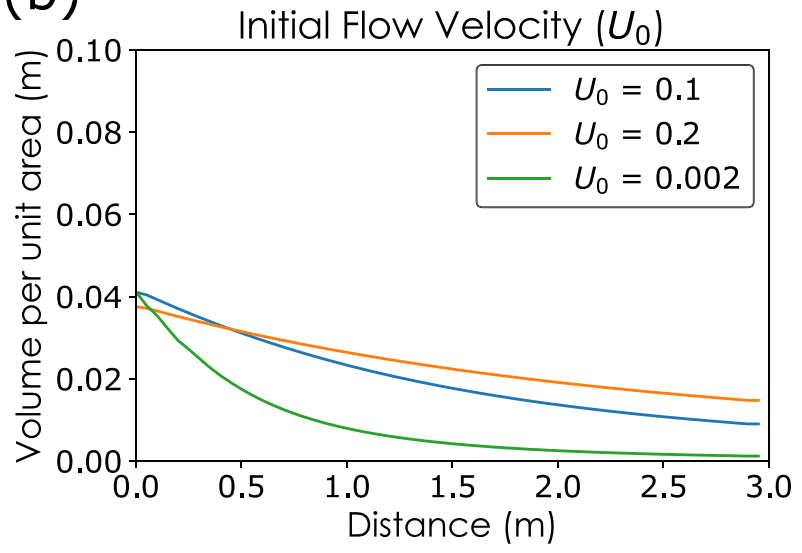

(d)

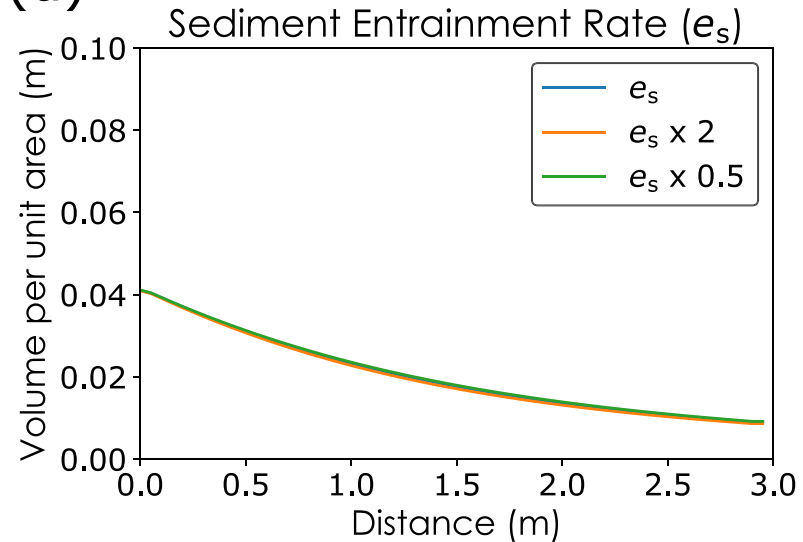

(f)

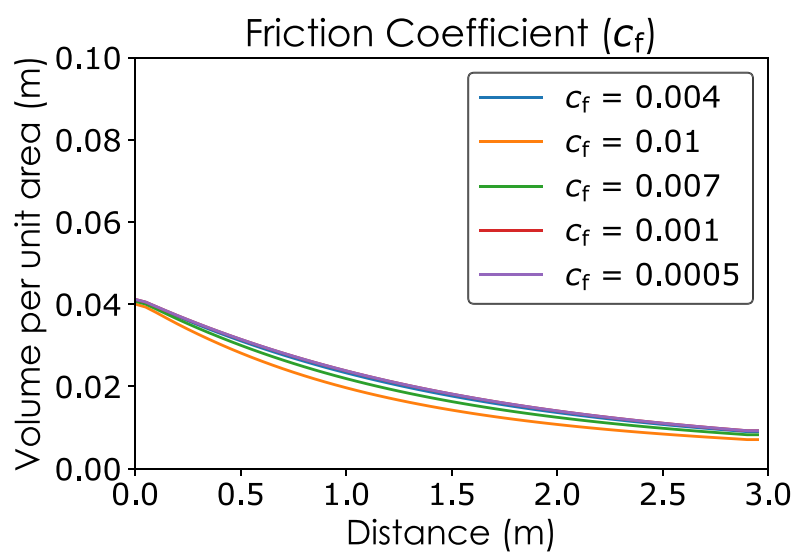

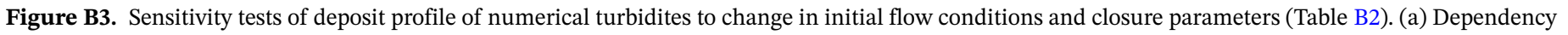

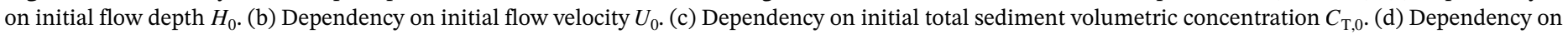
sediment entrainment rate $e_{\mathrm{s}}$. (e) Dependency on the ratio of near-bed to layer-averaged concentration $r_{\mathrm{o}}$. (f) Dependency on friction coefficient $c_{\mathrm{f}}$. 
(a)

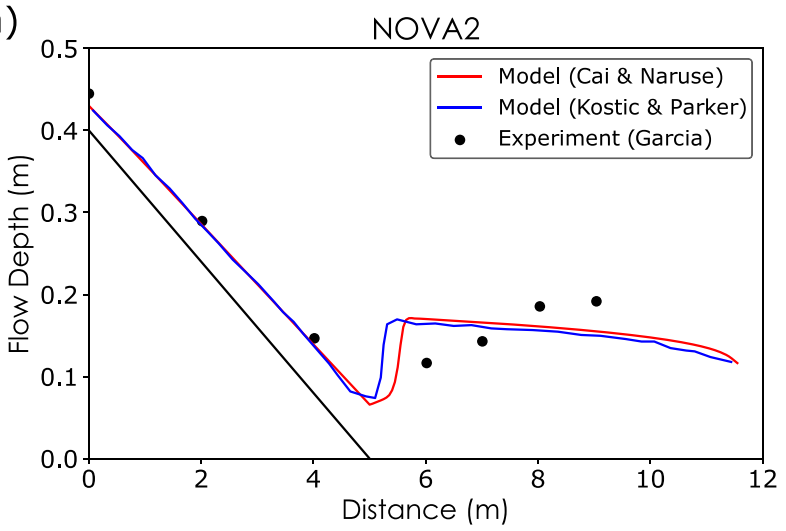

(b)

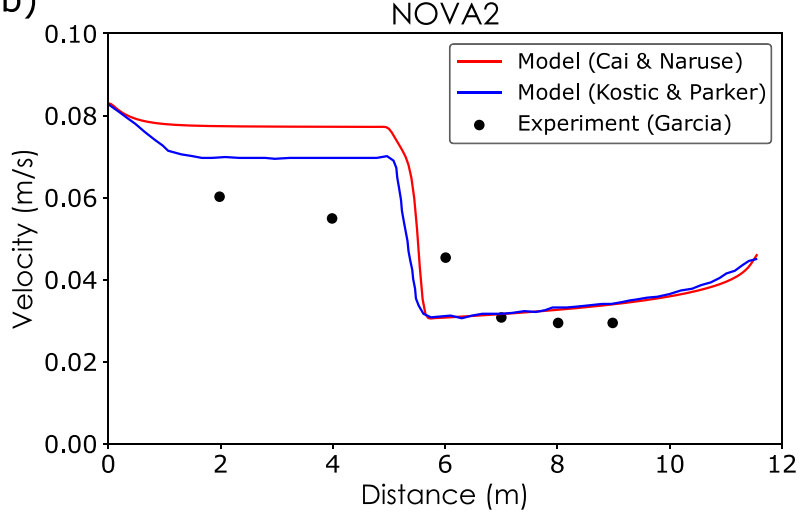

(c)

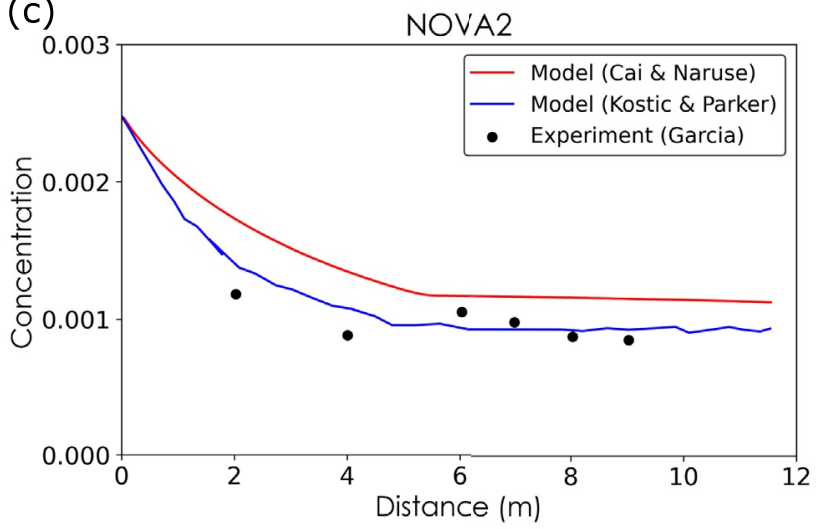

Figure B4. Forward model calculation results using initial flow conditions of experiment NOVA2 from García (1993). Plotted with experimental measurements from García (1993) and model results from Kostic and Parker (2006). (a) Flow depth profile. (b) Velocity profile. (c) Concentration profile.

The results of the sensitivity tests revealed that changes in the deposit profile occur when the initial flow conditions differ (Figure B3). The volume of the deposited sediment increased overall as $H_{0}$ increased (Figure B3a). The same trend was observed for $U_{0}$, and $C_{\mathrm{T}, 0}$ (Figures B3b and B3c). Among these three parameters, the amount of increase in the volume per unit area of deposit was greatest for $C_{\mathrm{T}, 0}$, and smallest for $U_{0}$ and $H_{0}$. Concerning model closure parameters, the resultant deposit profile showed almost no change for different values of entrainment coefficient $e_{\mathrm{s}}$ and $c_{\mathrm{f}}$ (Figures B3d and B3f). A slightly lower amount of deposition was observed for larger $e_{\mathrm{s}}$. A small increase in the amount of deposition was observed as $c_{\mathrm{f}}$ decreased (Figure B3f). The volume per unit area of deposit increased moderately when $r_{\mathrm{o}}$ increased. 
Table B2

Settings for Sensitivity Tests of Forward Model

\begin{tabular}{|c|c|c|c|c|c|c|}
\hline Case & $H_{0}(\mathrm{~m})$ & $U_{0}(\mathrm{~m} / \mathrm{s})$ & $C_{\mathrm{T}, 0}$ & $e_{\mathrm{s}}$ & $r_{\mathrm{o}}$ & $c_{\mathrm{f}}$ \\
\hline 1 & 0.15 & 0.1 & 0.01 & GP & 1.5 & 0.004 \\
\hline 2 & 0.3 & 0.1 & 0.01 & GP & 1.5 & 0.004 \\
\hline 3 & 0.05 & 0.1 & 0.01 & GP & 1.5 & 0.004 \\
\hline 4 & 0.15 & 0.2 & 0.01 & GP & 1.5 & 0.004 \\
\hline 5 & 0.15 & 0.02 & 0.01 & GP & 1.5 & 0.004 \\
\hline 6 & 0.15 & 0.1 & 0.02 & GP & 1.5 & 0.004 \\
\hline 7 & 0.15 & 0.1 & 0.001 & GP & 1.5 & 0.004 \\
\hline 8 & 0.15 & 0.1 & 0.01 & $\mathrm{GP} \times 2$ & 1.5 & 0.004 \\
\hline 9 & 0.15 & 0.1 & 0.01 & $\mathrm{GP} \times 0.5$ & 1.5 & 0.004 \\
\hline 10 & 0.15 & 0.1 & 0.01 & GP & 2.0 & 0.004 \\
\hline 11 & 0.15 & 0.1 & 0.01 & GP & 1.0 & 0.004 \\
\hline 12 & 0.15 & 0.1 & 0.01 & GP & 1.5 & 0.01 \\
\hline 13 & 0.15 & 0.1 & 0.01 & GP & 1.5 & 0.007 \\
\hline 14 & 0.15 & 0.1 & 0.01 & GP & 1.5 & 0.001 \\
\hline 15 & 0.15 & 0.1 & 0.01 & GP & 1.5 & 0.0005 \\
\hline
\end{tabular}

(a)

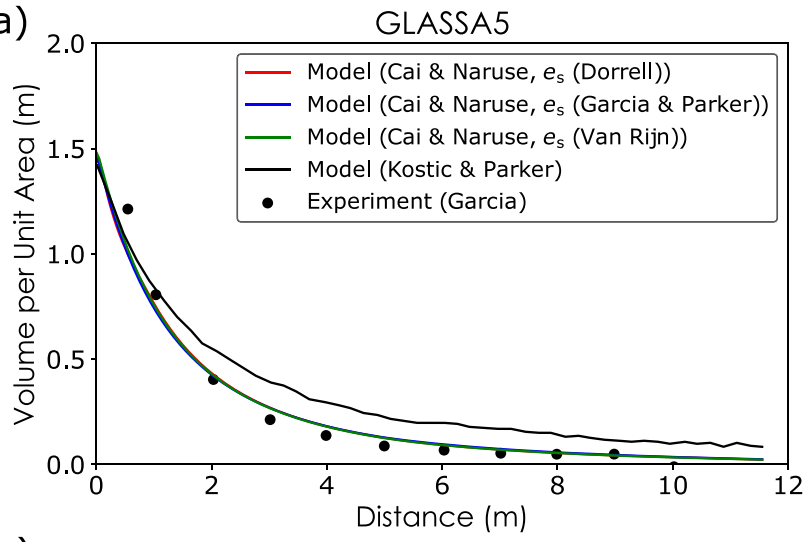

(b)

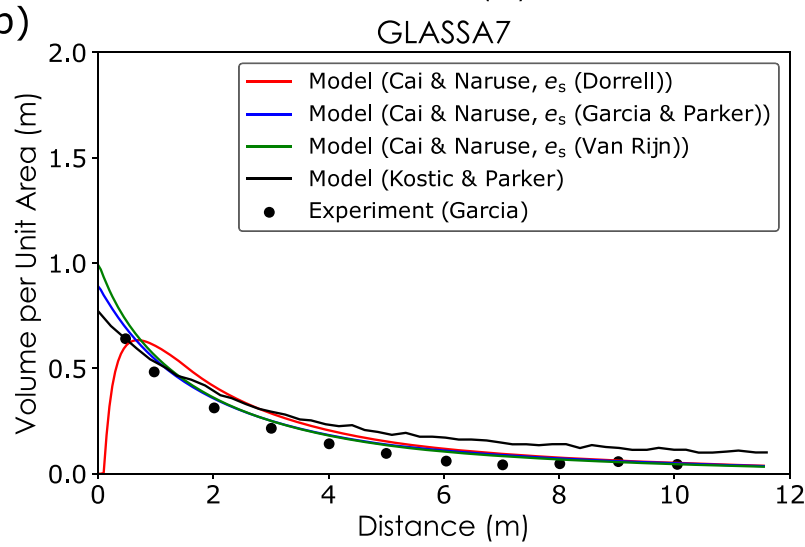

Figure B5. Forward model test of sensitivity to different entrainment functions using initial flow conditions of experiment GLASSA5 and GLASSA7 from (García, 1993). Plotted with experimental measurements from (García, 1993) and model results from Kostic and Parker (2006). (a) Deposit profile of GLASSA5 when calculated with different entrainment functions. (b) Deposit profile of GLASSA7 when calculated with different entrainment functions. 


\section{B3. Verification of Forward Model With Results From Previous Research}

We conducted calculations on experiment NOVA2 (García, 1993) under the same flow conditions and parameter settings as those used for modeling in Kostic and Parker (2006) to validate the numerical scheme and forward model implemented in this study. The resulting flow depth profile, velocity profile and concentration profile were compared with the model results from Kostic and Parker (2006) and the experiment data from García (1993) in Figure B4. The calculated flow depth profile showed an almost perfect match with that from Kostic and Parker (2006) (Figure B4a). The velocity profile was slightly higher than that of Kostic and Parker (2006) before the slope break, with close match for the values after the slope break (Figure B4b). The calculated concentration profile by the model in this study was slightly higher than that of Kostic and Parker (2006) (Figure B4c). The overall reconstruction by the model implemented in this study matched the results from the previous study by Kostic and Parker (2006).

\section{B4. Sensitivity of Forward Model to Different Entrainment Functions}

Calculations were conducted using the same initial flow conditions as those of experiments GLASSA5 and GLASSA7 (García, 1993) to test sensitivity of the implemented forward model to different entrainment functions. Three different entrainment functions were tested, including functions from van Rijn (1984), Garcia and Parker (1993), and Dorrell et al. (2018). The resulting deposit profiles are shown in Figure B5. Measurements from García (1993) and model results from Kostic and Parker (2006) are also shown for comparison. Figures B5a and B5b show that results from the model implemented in this study showed a closer match with the experimental measurements from García (1993), but the deposit profile showed almost no change with the change in entrainment function. A greater difference may be visible for a field scale simulation, but for experimental turbidity currents, the effect does not seem to be visibly large.

\section{Notation}

$\begin{array}{ll}\alpha_{1}, \alpha_{2} & \text { Parameters related to sediment entrainment } \\ B & \text { Bias } \\ c_{f} & \text { Friction coefficient } \\ C_{i} & \text { Layer-averaged volumetric concentration of suspended sediment of the ith grain size class } \\ \text { CI of } B & 95 \% \text { confidence interval of bias } \\ C_{s} & \text { Discharge coefficient } \\ C_{T} & \text { Layer-averaged total concentration of suspended sediment } \\ C_{*}^{v} & \text { The mid-value over the range in which the specific parameter was generated } \\ D_{i} & \text { Representative grain diameter of the ith grain-size class } \\ e_{\mathrm{Si}} & \text { Entrainment rate of sediment of the ith grain-size class into suspension } \\ e_{w} & \text { Entrainment rate of ambient water to flow } \\ F_{i} & \text { Volume fraction of the ith grain-size class in active layer } \\ g & \text { Gravitational acceleration } \\ H & \text { Flow depth } \\ L_{a} & \text { Active layer thickness } \\ M & \text { Flow discharge } \\ R & \text { Submerged specific density of sediment } \\ R_{\mathrm{fi}} & \text { Dimensionless particle fall velocity of the ith grain-size class } \\ R_{i} & \text { Bulk Richardson number } \\ \mathrm{Re}_{\mathrm{pi}} & \text { Particle Reynolds number of the ith grain-size class } \\ r_{o} & \text { Ratio of near-bed suspended sediment concentration to the layer-averaged concentration of } \\ s & \text { suspended sediment } \\ t & \text { Sample standard deviation } \\ T_{d} & \text { Time } \\ U & \text { Flow duration } \\ u_{*} & \text { Layer-averaged flow velocity } \\ & \text { Shear velocity } \\ & \end{array}$


$w_{i} \quad$ Settling velocity of a sediment particle of the ith grain-size class

$x \quad$ Streamwise distance

$\eta_{i} \quad$ Volume per unit area of bed sediment of the ith grain-size class

$\eta_{T} \quad$ Total volume per unit area of bed sediment

$\kappa \quad$ Parameter related to artificial viscosity

$\lambda_{p} \quad$ Porosity of bed sediment

$\mu \quad$ Dynamic viscosity of water

$\nu \quad$ Kinematic viscosity of water

$\rho \quad$ Density of water

$\theta \quad$ Angle of inclination of the base slope

\section{Data Availability Statement}

Doi for data and code is $10.5281 /$ zenodo.4780814.

\section{Acknowledgments}

This work was supported by the Sediment Dynamics Research Consortium (sponsored by JOGMEC, JX, INPEX, JAPEX) and JSPS KAKENH (20H01985). We would like to thank Enago (https://www.enago.jp/) for English language editing.

\section{References}

Abadi, M., Agarwal, A., Barham, P., Brevdo, E., Chen, Z., Citro, C., \& Zheng, X. (2015). TensorFlow: Large-scale machine learning on heterogeneous systems. Retrieved From http://tensorflow.org/

Altinakar, M., Graf, W., \& Hopfinger, E. (1996). Flow structure in turbidity currents. Journal of Hydraulic Research, 34(5), 713-718. https:// doi.org/10.1080/00221689609498467

Arai, K., Naruse, H., Miura, R., Kawamura, K., Hino, R., Ito, Y., et al. (2013). Tsunami-generated turbidity current of the 2011 tohoku-oki earthquake. Geology, 11(11), 1195-1198. https://doi.org/10.1130/g34777.1

Azpiroz-Zabala, M., Cartigny, M. J. B., Talling, P. J., Parsons, D. R., Sumner, E. J., Clare, M. A., et al. (2017). Newly recognized turbidity current structure can explain prolonged flushing of submarine canyons. Science Advances, 3(10), e1700200. https://doi.org/10.1126/ sciadv. 1700200

Baas, J. H., Van Dam, R. L., \& Storms, J. E. A. (2000). Duration of deposition from decelerating high-density turbidity currents. Sedimentary Geology, 136(1), 71-88. https://doi.org/10.1016/s0037-0738(00)00088-9

Biegert, E., Vowinckel, B., Ouillon, R., \& Meiburg, E. (2017). High-resolution simulations of turbidity currents. Progress in Earth and Planetary Science, 4(1), 33. https://doi.org/10.1186/s40645-017-0147-4

Bottou, L. (2010). Large-scale machine learning with stochastic gradient descent. In Y. Lechevallier, \& G. Saporta (Eds.), Proceedings of compstat'2010 (pp. 177-186). Heidelberg. Physica-Verlag HD. https://doi.org/10.1007/978-3-7908-2604-3_16

Bouma, A. H. (1962). Sedimentology of some flysch deposits: A graphic approach to facies interpretation. Elsevier.

Cantero, M. I., Balachandar, S., \& Garcia, M. H. (2007). High-resolution simulations of cylindrical density currents. Journal of Fluid Mechanics, 590, 437-469. https://doi.org/10.1017/s0022112007008166

Chikita, K. (1989). A field study on turbidity currents initiated from spring runoffs. Water Resources Research, 25(2), 257-271. https://doi. org/10.1029/WR025i002p00257

Daly, R. A. (1936). Origin of submarine canyons. American Journal of Science, 31(186), 401-420. https://doi.org/10.2475/ajs.s5-31.186.401

Davison, A. C., \& Hinkley, D. V. (1997). Bootstrap methods and their application. Cambridge University Press. https://doi.org/10.1017/ CBO9780511802843

Dietrich, W. E. (1982). Settling velocity of natural particles. Water Resources Research, 18(6), 1615-1626. https://doi.org/10.1029/ WR018i006p01615

Dorrell, R. M., Amy, L. A., Peakall, J., \& McCaffrey, W. D. (2018). Particle size distribution controls the threshold between net sediment erosion and deposition in suspended load dominated flows. Geophysical Research Letters, 45(3), 1443-1452. https://doi. org/10.1002/2017gl076489

Dorrell, R. M., Darby, S. E., Peakall, J., Sumner, E. J., Parsons, D. R., \& Wynn, R. B. (2014). The critical role of stratification in submarine channels: Implications for channelization and long runout of flows. Journal of Geophysical Research: Oceans, 119(4), 2620-2641. https://doi.org/10.1002/2014JC009807

Dorrell, R. M., Peakall, J., Sumner, E. J., Parsons, D. R., Darby, S. E., Wynn, R. B., et al. (2016). Flow dynamics and mixing processes in hydraulic jump arrays: Implications for channel-lobe transition zones. Marine Geology, 381, 181-193. https://doi.org/10.1016/j. margeo.2016.09.009

Falcini, F., Marini, M., Milli, S., \& Moscatelli, M. (2009). An inverse problem to infer paleoflow conditions from turbidites. Journal of Geophysical Research, 114, C10019. https://doi.org/10.1029/2009jc005294

Fukushima, Y., Parker, G., \& Pantin, H. M. (1985). Prediction of ignitive turbidity currents in Scripps Submarine Canyon. Marine Geology, 67(1), 55-81. https://doi.org/10.1016/0025-3227(85)90148-3

Garcia, M., \& Parker, G. (1991). Entrainment of bed sediment into suspension. Journal of Hydraulic Engineering, 117(4), 414-435. https:// doi.org/10.1061/(asce)0733-9429(1991)117:4(414)

Garcia, M., \& Parker, G. (1993). Experiments on the entrainment of sediment into suspension by a dense bottom current. Journal of Geophysical Research, 98(C3), 4793-4807. https://doi.org/10.1029/92jc02404

García, M. H. (1993). Hydraulic jumps in sediment-driven bottom currents. Journal of Hydraulic Engineering, 119(10), 1094-1117. https:// doi.org/10.1061/(ASCE)0733-9429(1993)119:10(1094)

Gibbs, R. J. (1974). A settling tube system for sand-size analysis. Journal of Sedimentary Petrology, 44(2), 583-588. https://doi. org/10.1306/74d72a9a-2b21-11d7-8648000102c1865d

Gunawan, H. P. (2015). Numerical simulation of shallow water Equations and related models (Theses, Université Paris-Est). Retrieved from https://tel.archives-ouvertes.fr/tel-01216642 
Heerema, C. J., Talling, P. J., Cartigny, M. J., Paull, C. K., Bailey, L., Simmons, S. M., et al. (2020). What determines the downstream evolution of turbidity currents? Earth and Planetary Science Letters, 532, 116023. https://doi.org/10.1016/j.epsl.2019.116023

Jameson, A., Schmidt, W., \& Turkel, E. (1981). Numerical solution of the euler Equations by finite volume methods using Runge Kutta time stepping schemes. In 14th fluid and plasma dynamics conference (p. 1259). https://doi.org/10.2514/6.1981-1259

Johnson, D. W. (1939). The origin of submarine canyons: A critical review of hypotheses. Columbia University Press.

Kostic, S., \& Parker, G. (2006). The response of turbidity currents to a canyon-fan transition: Internal hydraulic jumps and depositional signatures. Journal of Hydraulic Research, 44(5), 631-653. https://doi.org/10.1080/00221686.2006.9521713

Krizhevsky, A., Sutskever, I., \& Hinton, G. E. (2012). ImageNet classification with deep convolutional neural networks. In F. Pereira, C. J. C. Burges, L. Bottou, \& K. Q. Weinberger (Eds.), Advances in neural information processing systems (Vol. 25, pp. 1097-1105). Curran Associates, Inc.Retrieved from http://papers.nips.cc/paper/4824-imagenet-classification-with-deep-convolutional-neural-networks.pdf

Kuenen, P. H., \& Migliorini, C. I. (1950). Turbidity currents as a cause of graded bedding. The Journal of Geology, 58(2), 91-127. https:// doi.org/10.1086/625710

Lesshafft, L., Meiburg, E., Kneller, B., \& Marsden, A. (2011). Towards inverse modeling of turbidity currents: The inverse lock-exchange problem. Computers and Geosciences, 37(4), 521-529. https://doi.org/10.1016/j.cageo.2010.09.015

Liang, S., \& Srikant, R. (2016). Why deep neural networks for function approximation?. CoRR, abs/1610.04161. Retrieved from http://arxiv. org/abs/1610.04161

McIntosh, A. (2016). The jackknife estimation method.

Nakao, K., Naruse, H., \& Tokuhashi, S. (2020). Inverse analysis to reconstruct hydraulic conditions of non-steady turbidity currents: Application to an ancient turbidite of the kiyosumi formation of the Awa Group, Boso Peninsula, Central Japan (Vol. 10). EarthArXiv. Retrieved from https://eartharxiv.org/repository/view/1715/

Naruse, H. (2005). Usage and advantages of an application program "STube" for settling tube grain-size analysis. Journal of the Sedimentological Society of Japan, 62(62), 55-61. https://doi.org/10.4096/jssj1995.62.55

Naruse, H. \& Nakao, K. (2020). Inverse modeling of turbidity currents using an artificial neural network approach: Verification for field application. Earth Surface Dynamics Discussions, 1-27. https://doi.org/10.5194/esurf-2020-93

Naruse, H., \& Olariu, C. (2008). Hydraulic conditions of turbidity currents estimated by inverse analysis, In Fourth international conference on scour and erosion (pp. 591-593). Japanese Geotechnical Society.

Nielsen, M. A. (2015). Neural networks and deep learning. Determination Press.

Parker, G., Garcia, M., Fukushima, Y., \& Yu, W. (1987). Experiments on turbidity currents over an erodible bed. Journal of Hydraulic Research, 25(1), 123-147. https://doi.org/10.1080/00221688709499292

Parkinson, S. D., Funke, S. W., Hill, J., Piggott, M. D., \& Allison, P. A. (2017). Application of the adjoint approach to optimise the initial conditions of a turbidity current with the adjoint turbidity 1.0 model. Geoscientific Model Development, 10(3), 1051-1068. https://doi. org/10.5194/gmd-10-1051-2017

Paull, C. K., Talling, P. J., Maier, K. L., Parsons, D., Xu, J., Caress, D. W., et al. (2018). Powerful turbidity currents driven by dense basal layers. Nature Communications, 9(1), 4114. https://doi.org/10.1038/s41467-018-06254-6

Pradhan, B., Lee, S., \& Buchroithner, M. F. (2010). A GIS-based back-propagation neural network model and its cross-application and validation for landslide susceptibility analyses. Computers, Environment and Urban Systems, 34(3), 216-235. https://doi.org/10.1016/j. compenvurbsys.2009.12.004

Rogers, S. J., Fang, J. H., Karr, C. L., \& Stanley, D. A. (1992). Determination of lithology from well logs using a neural network. AAPG Bulletin, 76(5), 731-739.

Ruder, S. (2016). An overview of gradient descent optimization algorithms. CoRR, abs/1609.04747. Retrieved from http://arxiv.org/ abs/1609.04747

Rumble, J. R. (2018). Crc handbook of chemistry and physics (99th ed.). CRC Press.

Schmidhuber, J. (2015). Deep learning in neural networks: An overview. Neural Networks, 61, 85-117. https://doi.org/10.1016/j. neunet.2014.09.003

Simmons, S. M., Azpiroz-Zabala, M., Cartigny, M. J. B., Clare, M. A., Cooper, C., Parsons, D. R., et al. (2020). Novel acoustic method provides first detailed measurements of sediment concentration structure within submarine turbidity currents. Journal of Geophysical Research: Oceans, 125(5), e2019JC015904. https://doi.org/10.1029/2019JC015904

Talling, P. J., Allin, J., Armitage, D. A., Arnott, R. W. C., Cartigny, M. J. B., Clare, M. A., et al. (2015). Key future directions for research on turbidity currents and their deposits. Journal of Sedimentary Research, 85(2), 153-169. https://doi.org/10.2110/jsr.2015.03

Talling, P. J., Amy, L. A., \& Wynn, R. B. (2007). New insight into the evolution of large-volume turbidity currents: Comparison of turbidite shape and previous modelling results. Sedimentology, 54(4), 737-769. https://doi.org/10.1111/j.1365-3091.2007.00858.x

Talling, P. J., Masson, D. G., Sumner, E. J., \& Malgesini, G. (2012). Subaqueous sediment density flows: Depositional processes and deposit types. Sedimentology, 59(7), 1937-2003. https://doi.org/10.1111/j.1365-3091.2012.01353.x

Vangriesheim, A., Khripounoff, A., \& Crassous, P. (2009). Turbidity events observed in situ along the Congo submarine channel. Deep Sea Research Part II: Topical Studies in Oceanography, 56(23), 2208-2222. https://doi.org/10.1016/j.dsr2.2009.04.004

van Rijn, L. C. (1984). Sediment transport, part II: Suspended load transport. Journal of Hydraulic Engineering, 110(11), 1613-1641. https:// doi.org/10.1061/(ASCE)0733-9429(1984)110:11(1613)

Vestbøstad, T. M., Faltinsen, O. M., \& Kristiansen, D. (2007). Validation methods and benchmark tests for a 2-d CIP method applied to marine hydrodynamics. In The seventeenth international offshore and polar engineering conference.

Wang, Z., \& Bovik, A. C. (2009). Mean squared error: Love it or leave it? A new look at signal fidelity measures. IEEE Signal Processing Magazine, 26(1), 98-117. https://doi.org/10.1109/MSP.2008.930649

Weimer, P., \& Slatt, R. M. (2007). Introduction to the petroleum geology of deepwater settings. AAPG Studies in Geology, 57, 149-227. https://doi.org/10.1306/St571314

Xu, J. P., Noble, M. A., \& Rosenfeld, L. K. (2004). In-situ measurements of velocity structure within turbidity currents. Geophysical Research Letters, 31(9), 9311. https://doi.org/10.1029/2004gl019718

Yabe, T., Xiao, F., \& Utsumi, T. (2001). The constrained interpolation profile method for multiphase analysis. Journal of Computational Physics, 169(2), 556-593. https://doi.org/10.1006/jcph.2000.6625

Yang, H., Lu, M., \& Kumakura, T. (2016). A study on the water front in shallow water Equations. Journal of Japan Society of Civil Engineers, Ser. B1 Hydraulic Engineering, 72(4), I325-I330. https://doi.org/10.2208/jscejhe.72.I_325 